\title{
Lysophosphatidic acid-induced YAP/TAZ activation promotes developmental angiogenesis by repressing Notch ligand DII4
}

\author{
Daisuke Yasuda, ${ }^{1}$ Daiki Kobayashi, ${ }^{1}$ Noriyuki Akahoshi, ${ }^{1}$ Takayo Ohto-Nakanishi,, Kazuaki Yoshioka, ${ }^{2}$ Yoh Takuwa, ${ }^{2}$ \\ Seiya Mizuno, ${ }^{3}$ Satoru Takahashi, ${ }^{3}$ and Satoshi Ishii \\ 'Department of Immunology, Akita University Graduate School of Medicine, Akita, Japan. ²Department of Vascular Molecular Physiology, Kanazawa University Graduate School of Medicine, Ishikawa, Japan. \\ ${ }^{3}$ Laboratory Animal Resource Center, University of Tsukuba, Ibaraki, Japan.
}

\begin{abstract}
Lysophosphatidic acid (LPA) is a potent lipid mediator with various biological functions mediated through six G proteincoupled receptors (GPCRs), LPA1-LPA6. Previous studies have demonstrated that LPA-Ga12/Ga13 signaling plays an important role in embryonic vascular development. However, the responsible LPA receptors and underlying mechanisms are poorly understood. Here, we show a critical role of LPA4 and LPA6 in developmental angiogenesis. In mice, Lpa4;Lpa6 double-knockout (DKO) embryos were lethal due to global vascular deficiencies, and endothelial cell-specific (EC-specific) Lpa4;Lpa6-DKO retinas had impaired sprouting angiogenesis. Mechanistically, LPA activated the transcriptional regulators YAP and TAZ through LPA4/LPA6-mediated G $\alpha 12 / G \alpha 13-R h o-R O C K$ signaling in ECs. YAP/TAZ knockdown increased endothelial expression of the Notch ligand delta-like ligand 4 ( $D L L 4)$ that was mediated by $\beta$-catenin and Notch intracellular domain (NICD). Fibrin gel sprouting assay revealed that LPA4/LPA6, G $\alpha 12 / G \alpha 13$, or YAP/TAZ knockdown consistently blocked EC sprouting, which was rescued by a Notch inhibitor. Notably, the inhibition of Notch signaling also ameliorated impaired retinal angiogenesis in EC-specific Lpa4;Lpa6-DKO mice. Overall, these results suggest that the G $\alpha 12 / G \alpha 13-$ coupled receptors LPA4 and LPA6 synergistically regulate endothelial DII4 expression through YAP/TAZ activation. This could in part account for the mechanism of YAP/TAZ-mediated developmental angiogenesis. Our findings provide insight into the biology of GPCR-activated YAP/TAZ.
\end{abstract}

\section{Introduction}

Lysophosphatidic acid (LPA; 1- or 2-acyl-sn-glycerol-3-phosphate) is a bioactive lipid mediator that regulates cell migration, proliferation, morphological change, and the production of cytokines $(1,2)$. LPA is mainly produced by autotaxin (ATX), a secretory enzyme that converts lysophospholipids to LPA. Significant amounts of LPA have been detected in extracellular biological fluids such as serum, saliva, seminal fluid, and follicular fluid (3). LPA exerts its biological action through at least six $G$ protein-coupled receptors (GPCRs), LPA1-LPA6 (4-6). Depending on the functional cou-

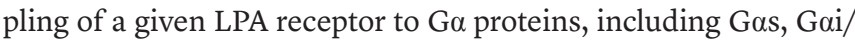

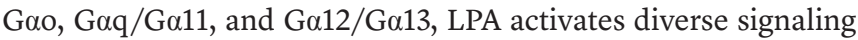
cascades $(4,6)$. Previously, we identified LPA4 (7) and LPA6 (8) and revealed that both receptors couple efficiently to $\mathrm{G} \alpha 12 / \mathrm{G} \alpha 13$ proteins $(8,9)$. These interactions activate Rho GTPase, which subsequently activates Rho-associated protein kinase I and II (ROCKI/II), leading to the induction of actin cytoskeletal tension (10). Likewise, LPA5-dependent Ga12/Ga13-Rho-ROCK activation was observed (11). Previous studies of various knockout (KO)

Conflict of interest: The authors have declared that no conflict of interest exists. Copyright: () 2019, American Society for Clinical Investigation.

Submitted: May 1, 2018; Accepted: July 16, 2019; Published: September 9, 2019.

Reference information: J Clin Invest. 2019;129(10):4332-4349.

https://doi.org/10.1172/JCl121955. mice lacking the LPA signaling components consistently showed that LPA-G $\alpha 12 / G \alpha 13$ signaling plays an important role in embryonic vascular development (12-14). For example, Atx-KO (12), Gna12;Gna13 double-KO (DKO) (13), and Rock1;Rock2-DKO (14) embryos displayed similar phenotypes of severely impaired blood vessel formation at E9.5-10.5. All of these embryos died by E11.5. However, the responsible LPA receptors and the underlying mechanisms are poorly understood. These studies are especially interesting to note because we detected mRNA expression of Lpa4 and Lpa6 in vascular endothelial cells (ECs) $(8,15,16)$. Furthermore, we found that Lpa4-KO embryos had pericardial effusion, subcutaneous hemorrhage, and dilated blood vessels with decreased mural cell coatings at different gestational stages (16). As a result of these abnormalities, however, only approximately 35\% of Lpa4KO mice were embryonic lethal. Therefore, another $\mathrm{G} \alpha 12 / \mathrm{G} \alpha 13-$ coupled LPA receptor such as LPA5 and/or LPA6 is assumed to cooperate with LPA4 in ECs for vascular development.

Blood vessel formation during mammalian embryonic development is a complex and tightly regulated process. In the past decade, the Notch signaling pathway has been shown to play a key role in vascular development (17). Notch signaling is an evolutionarily conserved pathway in multicellular organisms involved in numerous developmental processes, including cell fate determination, cellular differentiation, proliferation, survival, and apoptosis (18). Mice deficient in various Notch signaling components die in utero of severe 
vascular abnormalities (19). Delta-like ligand 4 (Dll4) is a transmembrane ligand for Notch receptors (Notch1 and Notch4), and it is expressed at a high level in arterial ECs (17). Dll4 regulates crucial processes during vascular growth, including EC sprouting and arterial specification (20). Previous studies revealed that Dll4 expression is regulated by various signaling pathways involving vascular endothelial growth factor-A (VEGF-A)/VEGF receptor-2 (VEGFR2), angiopoietin-1 (Ang-1)/Tie2, Wnt/ $\beta$-catenin, and Dll4/Notch itself (21-24). Haploinsufficiency of Dll4 in mice caused embryonic lethality due to severe vascular defects (25). Furthermore, even subtle changes in Dll4 expression impair vascular development $(26,27)$. However, the regulatory systems that fine-tune Dll4 expression during embryonic vascular development remain to be defined.

Yes-associated protein (YAP) and transcriptional coactivator with PDZ-binding motif (TAZ) are transcriptional regulators with similar functions (28). As primary downstream effectors of the Hippo signaling pathway, YAP/TAZ regulate signals related to tissue homeostasis, organ size, and cancer development (28). YAP/TAZ activity is limited through phosphorylation-dependent cytoplasmic retention by the Hippo pathway, while Rho activity and actin cytoskeleton tension exert a suppressive effect on YAP/ TAZ phosphorylation, promoting nuclear localization of YAP/ TAZ (29). A previous report showed that LPA-induced Ga12/Ga13 signaling activated YAP in HEK293 cells (30). Recently, several independent studies revealed that endothelial YAP/TAZ mediate sprouting angiogenesis (31-36). However, the biological significance of GPCR-induced YAP/TAZ activation is largely unknown.

Here, we address whether LPA4 and LPA6 synergistically function to contribute to developmental angiogenesis. We revealed that all Lpa4 $^{-/(Y)} \mathrm{Lpa6}^{-/-}$(termed hereafter Lpa4;Lpa6-DKO) embryos died by E11.5 due to global vascular deficiencies. Furthermore, EC-specific deletion of Lpa4 and Lpa6 genes resulted in impaired sprouting angiogenesis in the retina. In human umbilical vein endothelial cells (HUVECs), LPA-LPA4/LPA6-Ga12/ Ga13 signaling regulates endothelial DLL4 expression through YAP/TAZ activation. Notably, inhibition of this signaling pathway reduced sprouting angiogenesis in a $3 \mathrm{D}$ in vitro assay. We further demonstrated that a Notch inhibitor ameliorated both of the angiogenesis impairments in vivo and in vitro. Therefore, YAP/ TAZ are likely to mediate sprouting angiogenesis as downstream effectors of GPCR signaling. Our findings suggest that LPA4 and LPA6 are angiogenesis regulators that repress DLL4 expression by activating $\mathrm{YAP} / \mathrm{TAZ}$ in ECs.

\section{Results}

Lpa4;Lpa6-DKO mice are embryonic lethal due to vascular abnormalities. Among the progenies of more than 300 neonates obtained from Lpa6-heterozygous (Lpa6-Het) mouse intercrosses, an expected Mendelian distribution was observed (Supplemental Table 1; supplemental material available online with this article; https://oi.org/10.1172/JCI121955DS1), indicating that Lpa6KO mice were viable and healthy. However, we could not obtain Lpa4;Lpa6-DKO mice by Lpa4-KO;Lpa6-Het intercrosses (Supplemental Table 2). Further mating experiments revealed that the numbers of Lpa4-Het;Lpa6-KO and Lpa4-KO;Lpa6-Het mice were approximately $50 \%$ less than the value expected from Mendelian ratios (Supplemental Table 3). These results suggest that function- al cooperation between LPA4 and LPA6 is essential for embryonic development. Since Lpa4;Lpa5-DKO and Lpa5;Lpa6-DKO mice were born at the expected Mendelian ratios and had no obvious abnormalities (Supplemental Tables 4 and 5), LPA5 is unlikely to be involved in embryonic development.

To examine the roles of LPA4 and LPA6 in embryonic development, yolk sac and embryo proper were observed at various stages of gestation. At E8.5, both Lpa4;Lpa6-DKO yolk sac and embryo proper appeared normal (Supplemental Figure 1, A and B). However, at E9.5-10.5, almost all of Lpa4;Lpa6-DKO embryos proper had various morphological abnormalities, such as severe pericardial effusion (Figure $1 \mathrm{~A}$ and Supplemental Figure 1, B-D), axial turning abnormality (Supplemental Figure 1, B, E, and F), and developmental delay (Figure 1, A and B, and Supplemental Figure 1, B, G, and H). DKO yolk sacs lacked large blood vessels at E9.5-10.5, whereas WT yolk sacs had well-developed blood vessels (Figure 1B and Supplemental Figure 1, A, I, and J). We observed that all DKO embryos died by E11.5 (Supplemental Figure 1, B, K, and L, and Supplemental Table 6). Histological analysis showed that the endoderm and mesoderm of the yolk sacs were more widely separated in the DKO tissue (Figure 1C). Despite this vascular defect, erythrocytes were present in Lpa4;Lpa6-DKO yolk sacs (Figure 1C). Blood vessel-stained whole-mount embryos at E10.5 revealed that DKO embryos had enlarged dorsal aortae and poor vascular networks in the head and intersomitic regions, compared with WT embryos (Figure 1D). Furthermore, blood vessel-stained cross sections of embryos at E9.5 also revealed that DKO embryos had enlarged dorsal aortae and thinned neural tubes, compared with WT embryos (Figure 1E). These results strongly suggest that both LPA4 and LPA6 are indispensable for embryonic angiogenesis.

Endothelial LPA4 and LPA6 are involved in retinal angiogenesis. Previously, we detected mRNA expression of Lpa 4 and LPA 6 in vascular ECs $(8,16)$. To investigate the functional roles of endothelial LPA4 and LPA6, we generated a tamoxifen-inducible and EC-specific Lpa4/Lpa6 deletion mouse line by crossing Lpa4;Lpa6 double-floxed mutants with transgenic mice carrying Cdh5-CreER ${ }^{T 2}$ (ref. 37, Figure 2A, and Supplemental Figure 2A). The resulting $L p a 4^{f / f l(Y)} L p a 6^{f l / f l} C d h 5-C r e E R^{T 2}$ mice (termed hereafter Lpa4; $L p a 6^{i S E C}$ ) were treated with tamoxifen from P1 to P3. Allele-specific PCR confirmed recombination of both floxed alleles in the tails of Lpa4;Lpa6 ${ }^{\text {iSEC }}$ mice at P5 (Supplemental Figure 2B). At P5, we found that the radial expansion, EC area, and retinal blood vessel branching were significantly reduced in $L p a 4 ; L p a 6^{i \Delta E C}$ mice compared with those in control CreER $R^{T 2}$-negative $L p a 4^{f / f l(Y)} ; L p a 6^{f / f l}$ littermates (Figure 2, $\mathrm{C}$ and $\mathrm{F}-\mathrm{H}$ ). At the angiogenic front, sprouts of retinal vessels were significantly reduced in $L p a 4 ; L p a 6^{i \Delta E C}$ retina (Figure 2, D and I). Additionally, both the number and length of sprouting filopodia were significantly reduced in $L p a 4 ; L p a 6^{i \Delta E C}$ retina (Figure 2, E, J, and $\mathrm{K}$ ). These results suggested that endothelial LPA4 and LPA6 are critical for sprouting angiogenesis. No apparent body weight difference existed between control and Lpa4;Lpa6 ${ }^{\text {iLEC }}$ pups (Figure 2B), eliminating the possibility of a general growth defect due to endothelial LPA4/LPA6 deficiencies.

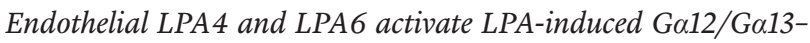
Rho-ROCK signaling. Vascular endothelial signaling pathways involved in LPA-induced angiogenesis remain largely undefined. 
A

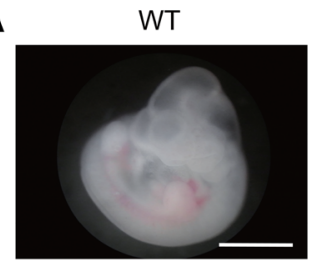

B
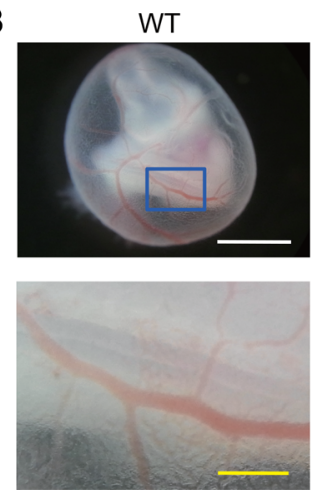

C
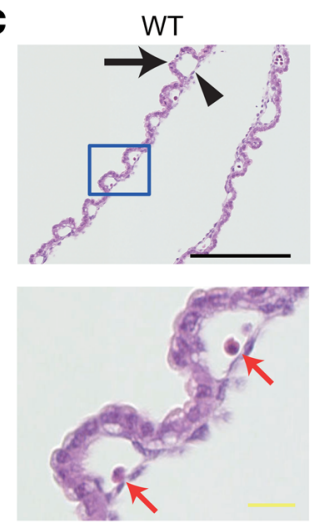

Lpa4:Lpa6 DKO
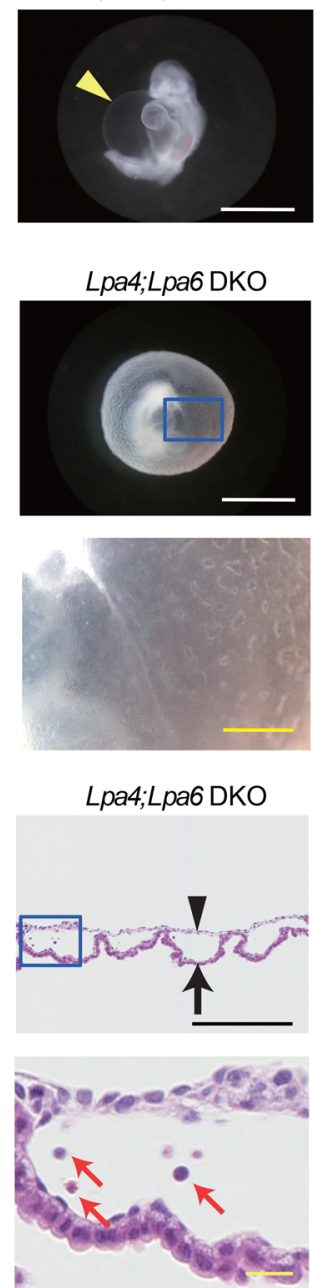

D
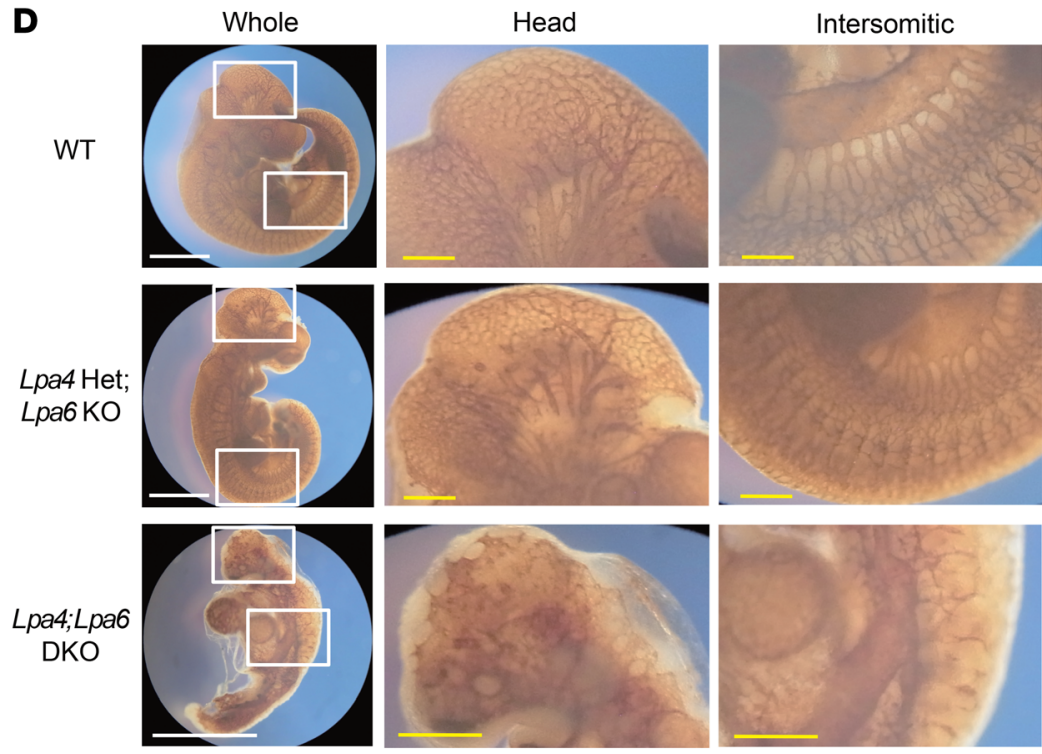

E
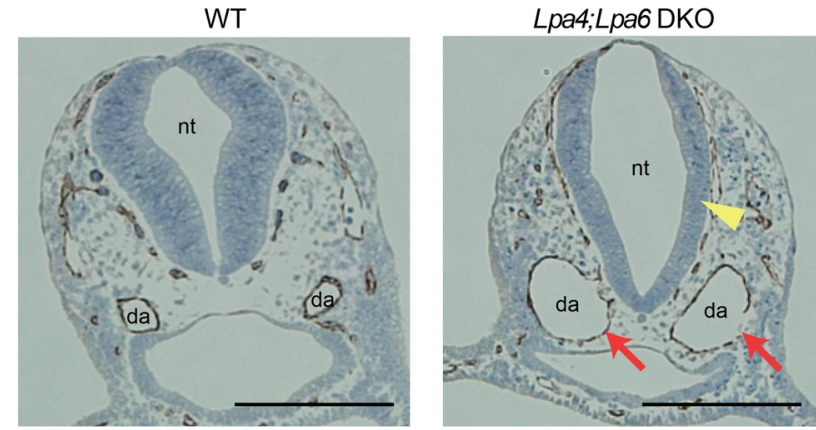

Figure 1. Lpa4;Lpa6-DKO embryo proper and yolk sac have vascular abnormalities. (A) Gross morphology of embryo proper at E10.5. Lpa4;Lpa6-DKO embryo proper had pericardial effusion as indicated by an arrowhead (WT, $n=11 ;$ DKO, $n=22$ ). Scale bars: $1 \mathrm{~mm}$. (B) Gross morphology of yolk sac at E10.5. The bottom panels show higher-magnification images of the boxed areas indicated in the top panels. Lpa4;Lpa6-DKO yolk sac lacked large and branched blood vessels (WT, $n=9$; DKO, $n=21$ ). White scale bars: $1 \mathrm{~mm}$; yellow scale bars: $200 \mu \mathrm{m}$. (C) H\&E-stained transverse section of yolk sac. The bottom panels show higher-magnification images of the boxed areas indicated in the top panels. Endoderm (arrow) and mesoderm (arrowhead) of $L p a 4 ; L p a 6$-DKO yolk sac were more widely separated (WT, $n=5$; DKO, $n=8$ ). Erythrocytes (red arrows) were present in DKO yolk sacs. Black scale bars: $1 \mathrm{~mm}$; yellow scale bars: $200 \mu \mathrm{m}$. (D) Vascular networks of embryo proper at E10.5. Blood vessels were stained with anti-PECAM-1 antibody. The middle and right panels show higher-magnification images of the boxed areas indicated in the left panels. Poor vascular networks in the head and intersomitic regions of $L p a 4$; Lpa6-DKO embryo proper were noted (WT, $n=4$; Lpa4-Het;Lpa6-KO, $n=4$; Lpa4;Lpa6-DKO, $n=5$ ). White scale bars: $1 \mathrm{~mm}$; yellow scale bars: $200 \mu \mathrm{m}$. (E) Transverse sections of the jugular area of embryo proper at E9.5. Blood vessels were stained with anti-PECAM-1 antibody. Enlargement of dorsal aortae (red arrows) and thinning of neural tube wall (yellow arrowhead) were remarkable in Lpa4;Lpa6-DKO embryo proper (WT, $n=5$; DKO, $n=6$ ). da, dorsal aorta; nt, neural tube. Scale bars: $200 \mu \mathrm{m}$. Representative images are shown.

HUVECs endogenously expressed LPA1, LPA4, and LPA6 mRNA (Figure 3A). We performed a serum response factor response element (SRF-RE) luciferase reporter assay, which detects the activation of the Ga12/Ga13-Rho-ROCK signaling pathway $(38,39)$. LPA increased the reporter activity, which was abolished by the ROCK inhibitor Y27632 and the siRNA-mediated knockdown of LPA4 and/or LPA6 but not by the LPA1/LPA3 antagonist Ki16425 (ref. 40; Figure 3, B and C; and Supplemental Figure 3A). These results suggested that LPA actually activates the Ga12/Ga13-Rho-ROCK signaling pathway through LPA4 and LPA6 in HUVECs. In contrast

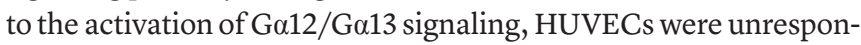
sive to LPA in terms of calcium influx (Figure 3D) and intracellular
cAMP level changes (Figure 3, E and F), which are cellular responses mediated by Gaq, Gai, and Gas proteins. Thus, LPA4 and LPA6 are unlikely to activate these Ga proteins strongly in HUVECs.

LPA reduces DLL4 expression in ECs through LPA4/LPA6-

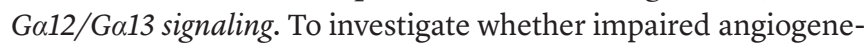
sis was associated with altered expression of angiogenesis-related genes, we performed quantitative reverse transcriptase PCR (RT-qPCR) screening for angiogenesis factors affected by LPA in HUVECs. Accordingly, we found several angiogenesis factors whose gene expression was changed by LPA (Figure 4A). Among them, we focused on the important angiogenesis factor DLL4, which was remarkably repressed by LPA (Figure 4B). Consis- 
A

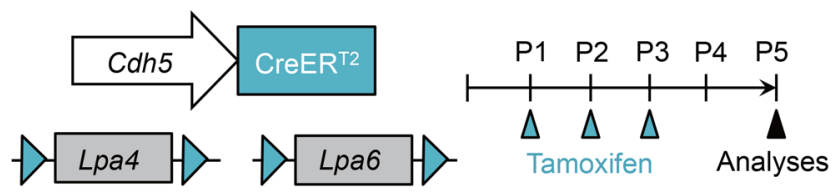

C

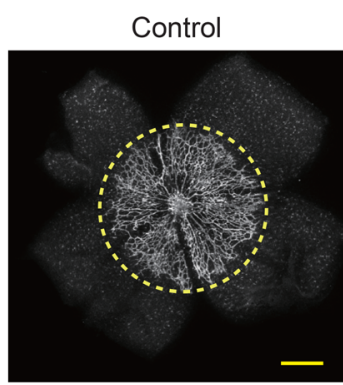

D

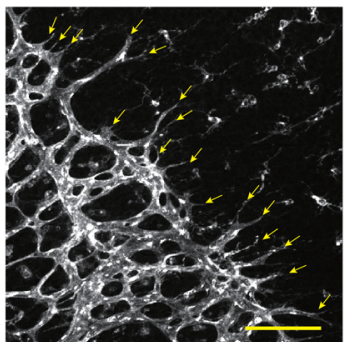

E

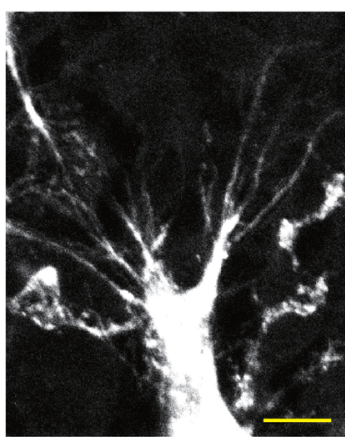

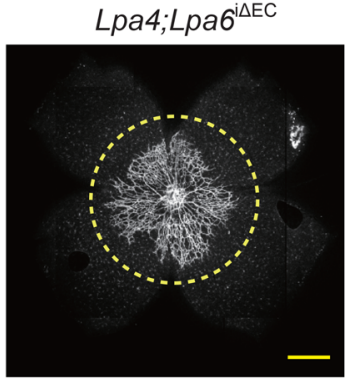
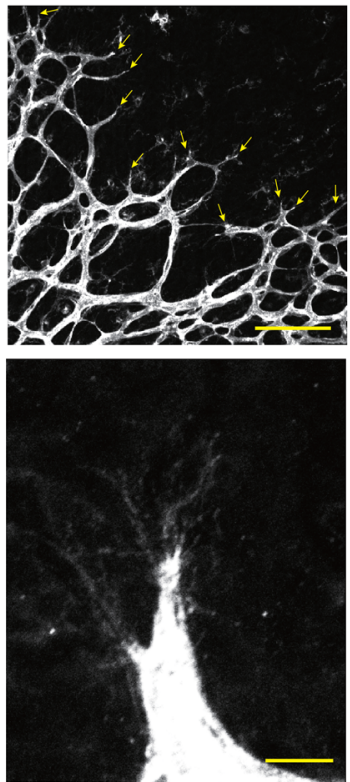

B

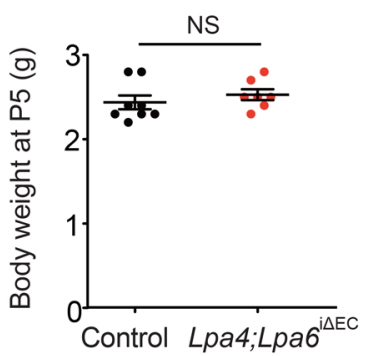

$\mathbf{F}$
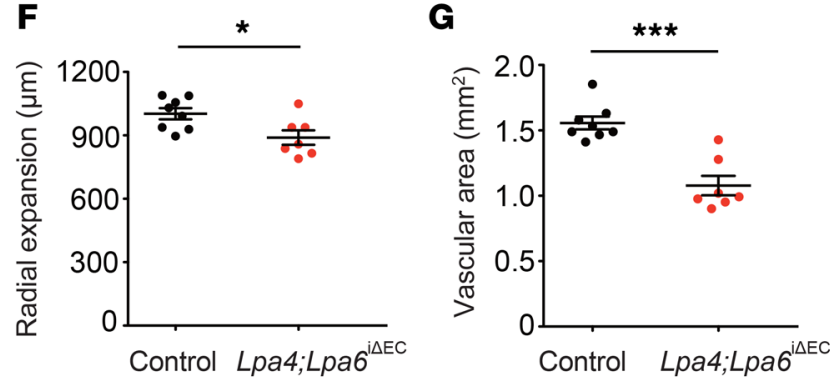

H

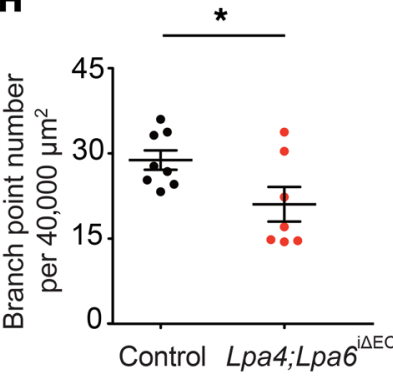

I

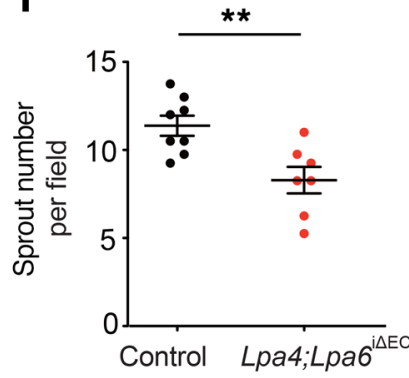

J

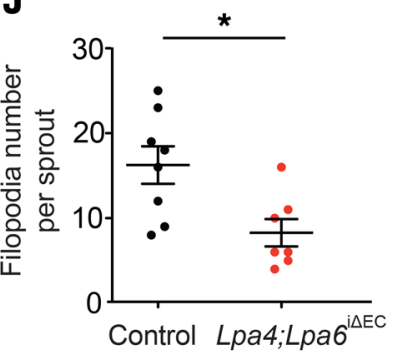

$\mathbf{K}$

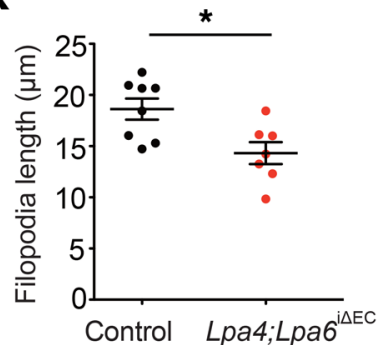

Figure 2. Endothelial LPA4 and LPA6 are involved in retinal angiogenesis. (A) Diagram for EC-specific $L p a 4 / L p a 6$ deletion in the retinal vessels from P1 and their analyses at P5 using $L p a 4^{f / / f(Y)} ; L p a 6^{f / / f l} ; C d h 5-C r e E R^{T 2}\left(L p a 4 ; L p a 6^{i \Delta E C)}\right.$ and Cdh5-CreER ${ }^{T 2}$-negative Lpa $4^{f / / f(Y)} ; L p a 6^{f / / f l}$ (control) littermates. (B) Body weight of $L p a 4 ; L p a 6^{i \Delta E C}$ and control littermates at P5. (C) Representative confocal images of retinas of $L p a 4 ; L p a 6^{i \Delta E C}$ and control littermates. Vessels were stained with isolectin B4. Yellow dashed circles indicate the front edge of radial expansion of control retina. Scale bars: $500 \mu \mathrm{m}$. (D) Representative confocal images of retinas at the edge of the angiogenic growth front. Sprouts are indicated by yellow arrows. Scale bars: $100 \mu \mathrm{m}$. (E) Representative high-magnification confocal images of retinas at the edge of the angiogenic growth front. Scale bars: $10 \mu \mathrm{m}$. (F-K) Quantitative analyses of retinal vessel expansion (F), vascular area (G), branch point number (H), sprout number (I), filopodia number (J), and filopodia length (K). Each dot represents the value of a mouse. Horizontal lines represent mean $\pm \mathrm{SEM}\left(n=8\right.$ control and $7 \mathrm{Lpa4}$; $L p a 6^{\mathrm{i} A E C}$ mice). One retina was analyzed per mouse. ${ }^{*} P<0.05,{ }^{* *} P<0.01,{ }^{* * *} P<0.001$, 2-tailed unpaired Student's $t$ test.

tent with RT-qPCR analysis, LPA treatment reduced the protein expression level of DLL4 in HUVECs in a time-dependent manner (Figure 4C). Conversely, LPA4/LPA6 siRNAs and also Ga12/ Ga13 siRNAs increased the mRNA and protein expression levels of DLL4 (Figure 4, D and E, and Supplemental Figure 3, A-C). Likewise, Rho inhibitor I and Y27632, but not Ki16425, increased DLL4 expression. Rho inhibitor I and Y27632, as well as LPA4/ LPA6 siRNAs, consistently abolished the negative effect of LPA on DLL4 expression (Figure 4, F-H). Transient overexpression of LPA4 and/or LPA6 considerably decreased DLL4 mRNA and pro- tein expression, while LPA1, LPA2, LPA3, and LPA5 showed little or no effect on DLL4 expression (Supplemental Figure 4, A-C). Additionally, the transient overexpression of LPA4 and/or LPA6 also decreased the mRNA expression of HES1, a Notch target gene (Supplemental Figure 4D). Consistent results were obtained from the lung ECs of $L p a 4 ; L p a 6^{\triangle E C}$ mice, which were obtained by crossing of Lpa4;Lpa6 double-floxed mutants with transgenic mice carrying Tie2-Cre (Supplemental Figure 2, C and D); Lpa4/Lpa6 deletion in the ECs led to increased mRNA expression of Dll4 and the Notch target gene Hey1 (Figure 4, I and J, and Supplemental Figure 
A

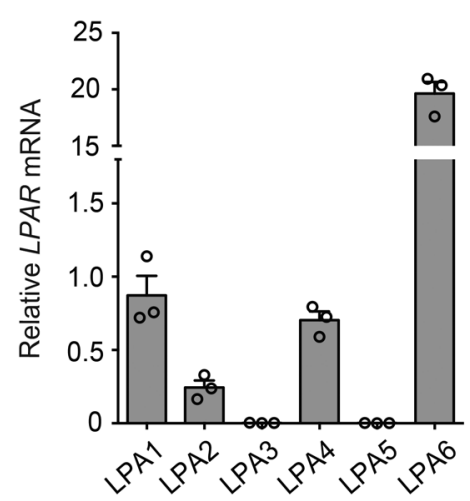

D

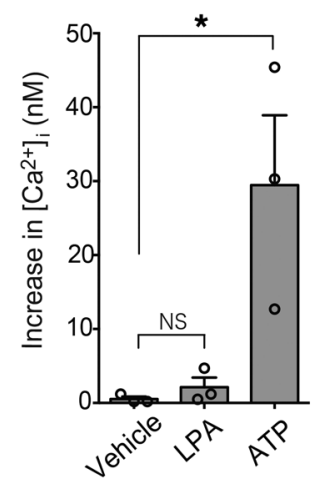

B

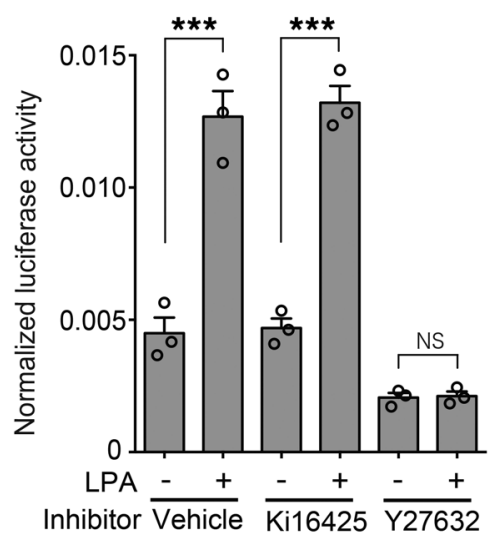

C

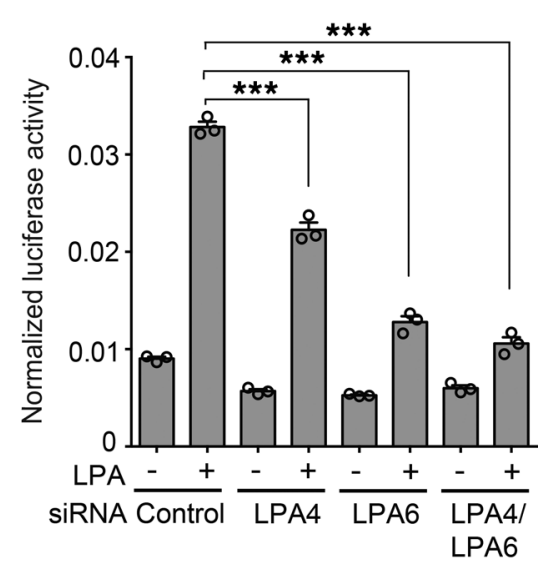

$\mathbf{F}$

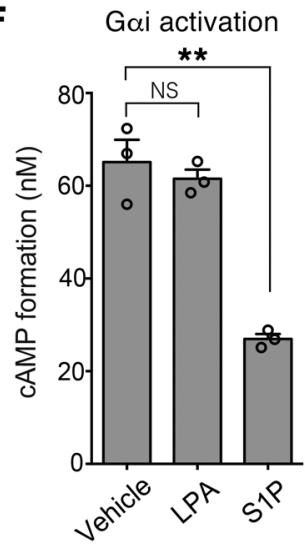

Figure 3. LPA4 and LPA6 in HUVECs mediate LPA-induced $\mathbf{G} \alpha \mathbf{1 2} / \mathbf{G} \alpha \mathbf{1 3}$ activation. (A) Expression of mRNA for LPA receptors was detected by RT-qPCR. (B) SRF-RE-Luc reporter assay was performed to detect G $\alpha 12 / G \alpha 13-R$ ho activation. LPA (10 $\mu \mathrm{M}, 6$ hours) increased the reporter activity, which was attenuated by the ROCK inhibitor Y27632 (10 $\mu \mathrm{M}, 10$ minutes pretreatment) but not by the LPA1/LPA3 antagonist Ki16425 (10 $\mu \mathrm{M}, 10$ minutes pretreatment). (C) LPA-induced SRF-RE-Luc activity was attenuated by LPA4/LPA6 siRNAs (12.5 nM each, 48 hours pretreatment). (D) Intracellular calcium influx assay was performed to detect $G \alpha q$ activation. A representative trace of changes in intracellular $\mathrm{Ca}^{2+}$ concentration $\left(\left[\mathrm{Ca}^{2+}\right]_{i}\right)$ is shown on the right. HUVECs were unresponsive to LPA $(10 \mu \mathrm{M})$. Adenosine 5'-triphosphate (ATP; $10 \mu \mathrm{M}$ ) was used as a positive control, because ATP evokes calcium influx via P2Y receptors that predominantly couple with G $\alpha q$. (E) cAMP assay was performed to detect Gas activation. No significant increase in cAMP level was observed in HUVECs treated with LPA $(10 \mu \mathrm{M})$. Forskolin $(20 \mu \mathrm{M})$ was used as a positive control. (F) cAMP assay was performed to detect Gai activation. Inhibitory effect of LPA $(10 \mu \mathrm{M})$ on cAMP accumulation stimulated by forskolin $(20 \mu \mathrm{M})$ was not observed in HUVECs. Sphingosine-1-phosphate (S1P; $100 \mathrm{nM})$ was used as a positive control. Data are mean \pm SEM of triplicates. ${ }^{*} P<0.05$, ${ }^{* *} P<0.01$, ${ }^{* *} P<0.001$, 1-way ANOVA followed by Tukey's multiple-comparisons test.

5). In subsequent immunohistological analysis of the retinas, we detected increased Dll4 protein expression at the angiogenic front of tamoxifen-administered $L p a 4 ; L p a 6^{i \Delta E C}$ mice, as compared with expression levels in Cre-negative control mice (Figure 5A). In contrast, Dll4 expression at the artery was unaffected by endothelial Lpa4/Lpa6 deletion (Figure 5B). These findings indicate that the LPA-LPA4/LPA6-Ga12/Ga13-Rho-ROCKI/II signaling pathway prevents DLL4 expression in ECs (Figure 4K).

Inactivation of YAP and TAZ increases DLL4 expression in ECs. The major function of Rho is to regulate cellular actin dynamics (28). We therefore examined whether changes in the actin cytoskeleton contribute to DLL4 expression. When HUVECs were treated with the actin-disrupting agent latrunculin A, the suppressive effects of LPA on DLL4 expression were blocked. In addition, latrunculin A treatment per se remarkably increased the mRNA and protein expression levels of DLL4 (Figure 6, A and B). Correspondingly, the mRNA expression level of EFNB2 was increased by latrunculin A (Figure 6C). To address the molecular mechanisms underlying the LPA-induced DLL4 suppression, we focused on the transcription coactivators YAP and TAZ, because an important role of actin polymerization has been reported in LPA-induced YAP activation (30). As expected, siRNA-mediated knockdown of YAP and TAZ drastically increased the mRNA and protein expression levels of DLL4 (Figure 6, D and E, and Supplemental Figure 3, D and E). The YAP/TAZ knockdown also increased the mRNA expression levels of HEY1, HEY2, HES1, and EFNB2 (Figure 6F). For the similar purpose of inactivating YAP/TAZ, HUVECs were subjected to YAP inhibitor treatment with ivermectin (ref. 41 and Figure 6, G and H), serum starvation (ref. 30 and Figure 6, I and $\mathrm{J}$ ), or overconfluent culture conditions (ref. 34 and Figure 6, K and L). Taken together, we consistently observed the increased mRNA and protein expression of DLL4 by YAP/TAZ inactivation.

LPA activates YAP through LPA4 and LPA6 in ECs. As described in the last section, serum-starved and overconfluent 


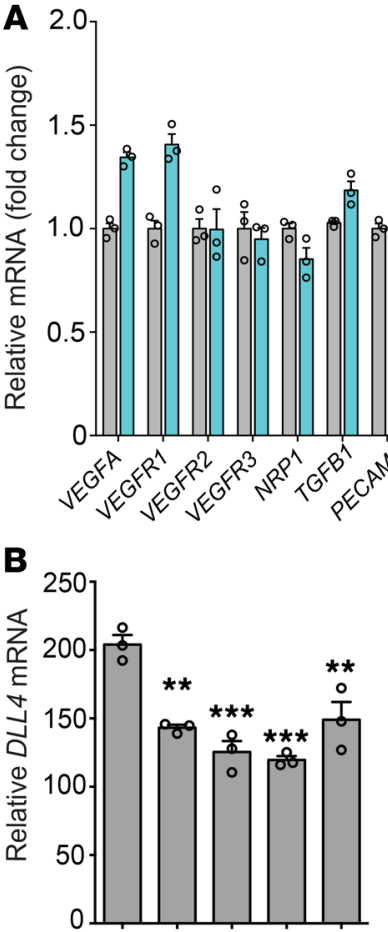

Vehicle

$\square$ LPA 3h
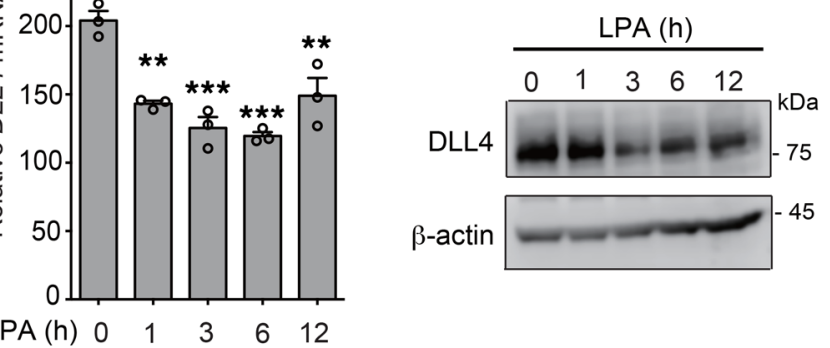

D

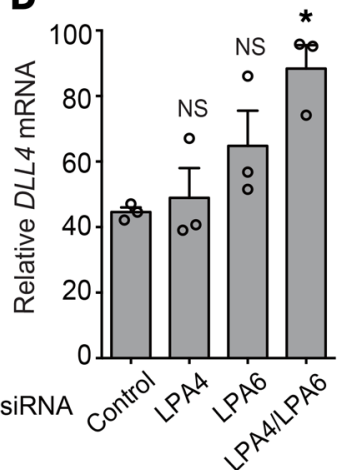

E

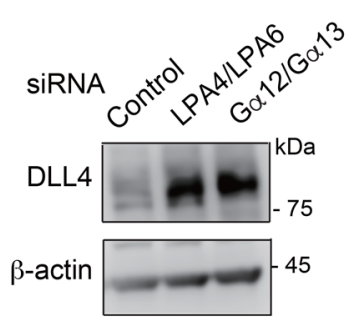

$\mathbf{F}$
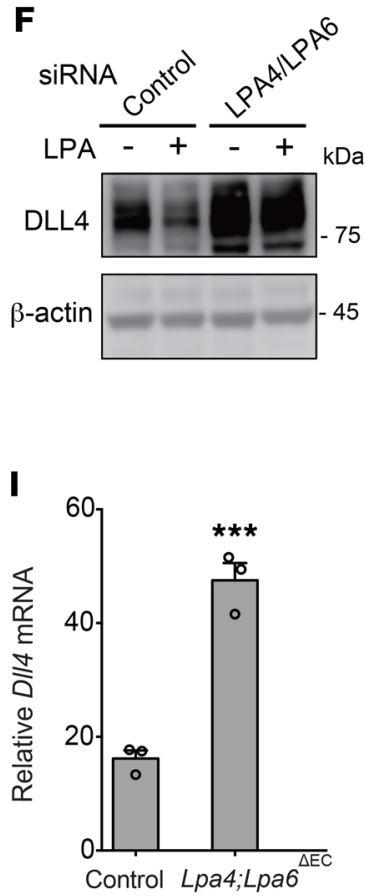

G

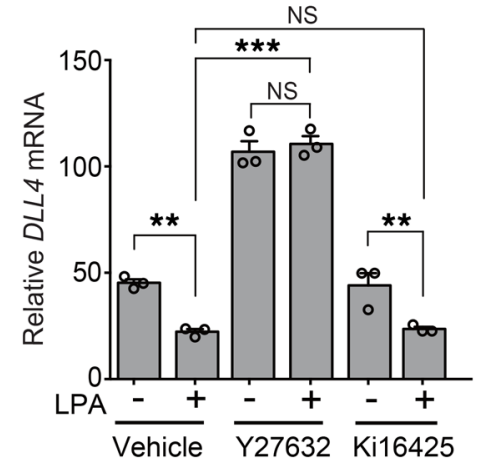

J

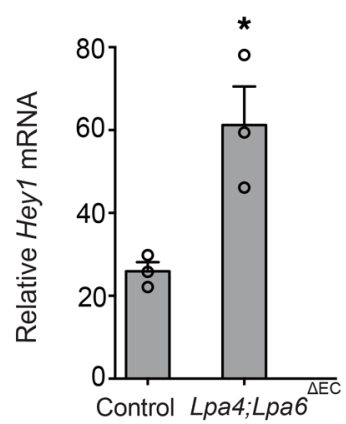

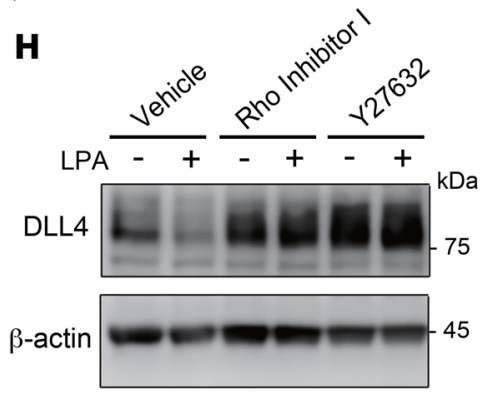

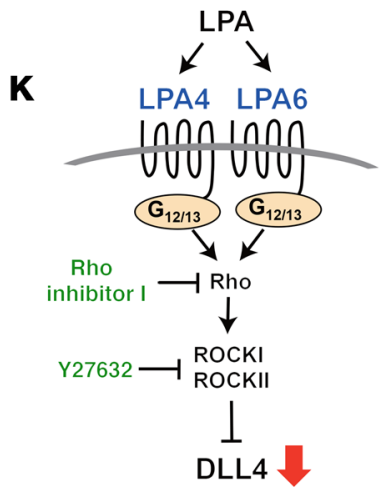

Figure 4. DLL4 expression is negatively regulated by LPA through LPA4/LPA6-Ga12/Ga13-Rho-ROCK signaling in ECs. (A) LPA (10 $\mu$ M, 3 hours) affected mRNA expression of various angiogenesis-related genes in serum-starved HUVECs. (B and C) LPA (10 $\mu$ M) reduced mRNA (B) and protein (C) expression of DLL4 in a time-dependent manner in serum-starved HUVECs. (D) LPA4 and/or LPA6 siRNAs increased mRNA expression of DLL4. (E) LPA4/LPA6 and Ga12/Ga13 siRNAs increased protein expression of DLL4. (F) LPA4/LPA6 siRNAs increased protein expression of DLL4 and abolished the suppressive effect of LPA (10 $\mu$ M, 3 hours) in serum-starved HUVECs. (G) Y27632 (10 $\mu \mathrm{M}, 10$ minutes pretreatment) but not Ki16425 (10 $\mu \mathrm{M}, 10$ minutes pretreatment) increased mRNA expression of DLL4 and abolished the suppressive effect of LPA ( $10 \mu \mathrm{M}$, 3 hours) in serum-starved HUVECs. (H) Rho inhibitor I ( $0.5 \mu \mathrm{g} / \mathrm{mL}, 6$ hours pretreatment) and Y27632 (10 $\mu \mathrm{M}, 10$ minutes pretreatment) increased protein expression of DLL4 and abolished the suppressive effect of LPA (10 $\mu$ M, 3 hours) in serum-starved HUVECs. (I and J) Mouse

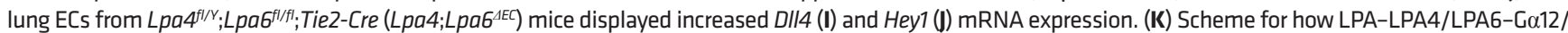
Ga13-Rho-ROCK signaling suppresses DLL4 expression. Data are mean \pm SEM of triplicates. ${ }^{*} P<0.05,{ }^{* *} P<0.01,{ }^{* * *} P<0.001,1$-way ANOVA followed by Dunnett's (B and D) or Tukey's multiple-comparisons test (C) or 2-tailed unpaired Student's $t$ test (I and J). HUVECs were serum-starved for 8 hours or transfected with siRNAs for 96 hours. Unprocessed original scans of Western blots are shown in Supplemental Figure 12. 
A

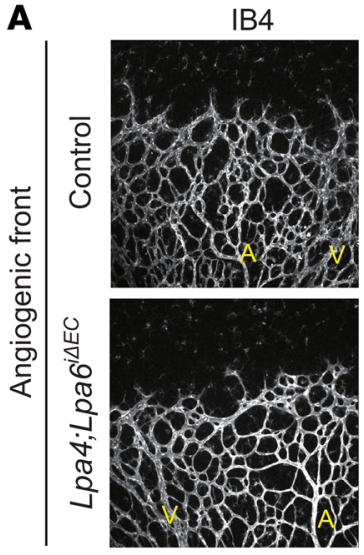

B

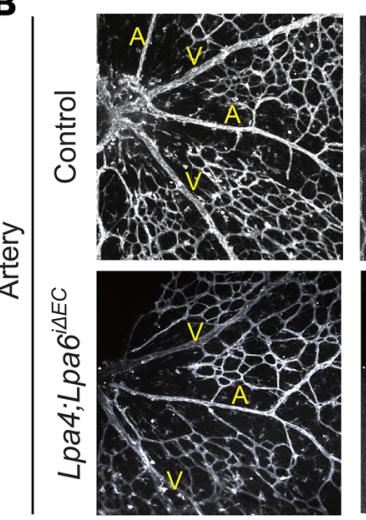

DII4
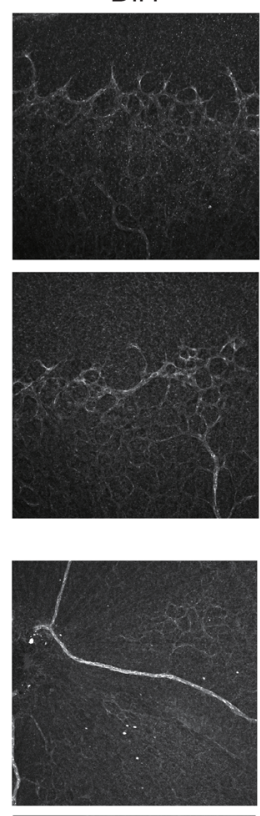

(4)
|B4-D||4
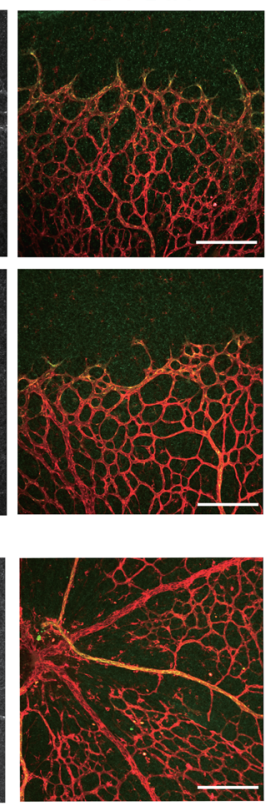

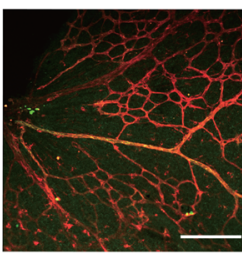

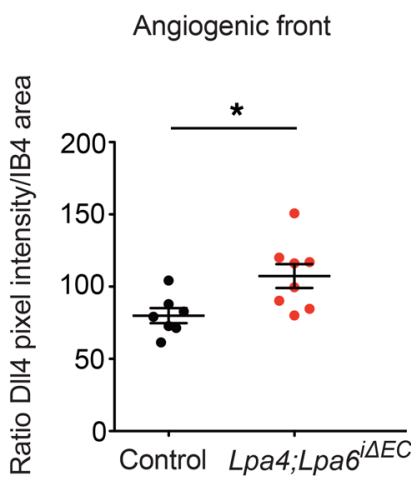

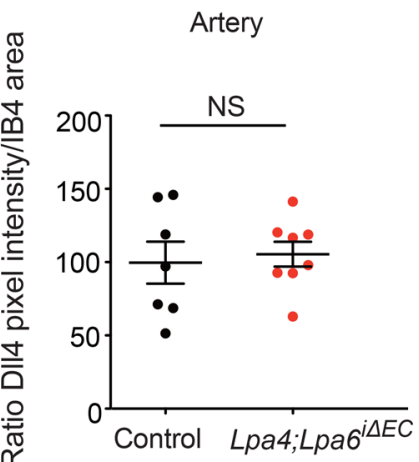

Figure 5. Endothelial-specific Lpa4/Lpa6 deletion increases Dll4 expression at the angiogenic front. (A and B) Representative confocal images and quantitative analyses of DII4 expression in the angiogenic front (A) and artery (B) of P5 retinal vessels from Lpa4;Lpa6 ${ }^{\text {iLEC }}$ and control littermates. Retinas were costained with isolectin B4 (IB4; red) and DII4 (green). Scale bars: $100 \mu \mathrm{m}$. Arteries $(A)$ and veins $(V)$ are indicated. Horizontal lines represent mean \pm SEM ( $n=7$ control and 8 Lpa4;Lpa $6^{\text {iSEC }}$ mice). One retina was analyzed per mouse. ${ }^{*} P<0.05,2$-tailed unpaired Student's $t$ test. culture conditions localized YAP/TAZ predominantly in the cytoplasm of HUVECs, and this was mediated by their phosphorylation $(28,34)$. These conditions allowed us to clearly observe the nuclear translocation of YAP/TAZ in response to LPA in HUVECs (Figure 7A). siRNA-mediated knockdown of LPA4 and/or LPA6 inhibited the LPA-induced dephosphorylation (Figure 7B) and nuclear localization (Figure 7A) of YAP. Y27632 also inhibited YAP nuclear localization induced by LPA (Figure 7C). In line with these results, the LPA4 and/or LPA6 knockdown attenuated the increase in mRNA expression of YAP target genes, such as CTGF and CYR61, by LPA (Supplemental Figure 6A). Y27632, but not Ki16425, attenuated the LPA-induced CTGF and CYR61 mRNA expression (Supplemental Figure 6B). In mouse lung ECs, LPA-induced nuclear localization of YAP was consistently suppressed by Lpa4/Lpa6 deletion or Y27632 treatment (Figure 7D). In contrast to the prominent nuclear localization of YAP at the retinal angiogenic front of control mice, YAP was mainly localized within cytoplasm in tamoxifen-administered Lpa4;Lpa $6^{\text {iAEC }}$ mice (Figure 7E). Together, these results suggest that endothelial LPA4 and LPA6 are responsible for the LPA-G $\alpha 12 / \mathrm{G} \alpha 13-\mathrm{Rho}-\mathrm{ROCKI} / \mathrm{II}$ signaling that leads to YAP activation.

LPA4/LPA6-mediated signaling functionally modulates angiogenesis by inhibiting Dll4 expression in mice. To determine the functional roles of LPA4 and LPA6 in ECs, we performed a 3D bead sprouting assay with HUVECs. This assay recapitulates all the major steps of angiogenesis seen in vivo (42). LPA4/LPA6, Ga12/Ga13, or YAP/TAZ knockdown by gene-specific siRNA consistently suppressed the length and number of sprouts from the HUVEC-coated beads (Figure 8, A-C). Furthermore, knockdown of LPA4/LPA6 also blocked EC proliferation (Supplemental Figure 7). When we inhibited Notch receptor cleavage and release of Notch intracellular domain (NICD) using the $\gamma$-secretase inhibitor $\quad N$-[N-(3,5-difluorophenacetyl)-L-alanyl]-(S)-phenylglycine $t$-butyl ester (DAPT) (refs. 20, 43, and Supplemental Figure 8), the sprouting defects were significantly rescued (Figure 8, A-C). DAPT also significantly ameliorated the sprouting defects of Y27632-treated HUVECs (Figure 8, D-F). Consistent results were obtained from the mouse lung ECs (Figure 8, G-I). Additionally, the sprouting defects of gene-specific siRNA- or Y27632-treated HUVECs were significantly rescued by concomitant DLL4 knockdown (Supplemental Figure 9).

In addition to the pharmacological efficacy in vitro, DAPT exerted a significant ameliorating effect on the impaired retinal angiogenesis of $L p a 4 ; L p a 6^{i \mathrm{iEC}}$ mice (Figure 9), while exerting no effect on body weight. Thus, the results observed after the pharmacological inhibition of Notch strongly suggest that the reduced angiogenesis in $L p a 4 ; L p a 6^{\mathrm{i} E C}$ retina was indeed a consequence of increased Dll4 expression.

Nuclear YAP suppresses DLL4 expression in a TEA domainindependent manner. It has been reported that the transcriptional corepressor function of YAP requires TEA domain (TEAD) transcription factors (44). To reveal the involvement of TEAD transcription factors in YAP-induced DLL4 suppression, we first tested whether siRNAs targeting TEAD1-4 affect DLL4 expression in HUVECs. TEAD1-4 knockdown did not alter DLL4 expression (Figure 10, A and B, and Supplemental Figure 10A); however, knockdown of these transcription factors did significantly reduce the expression of CTGF and CYR61 mRNA 


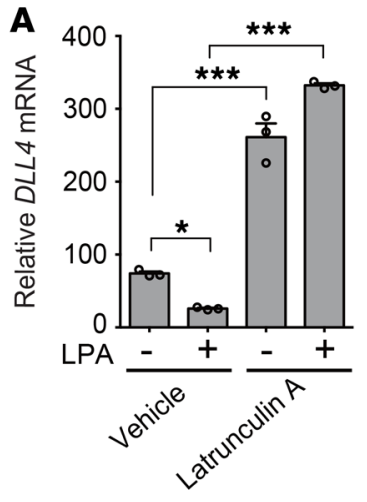

B

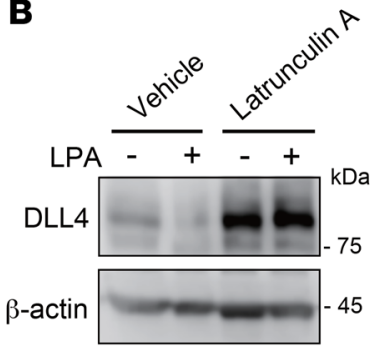

C
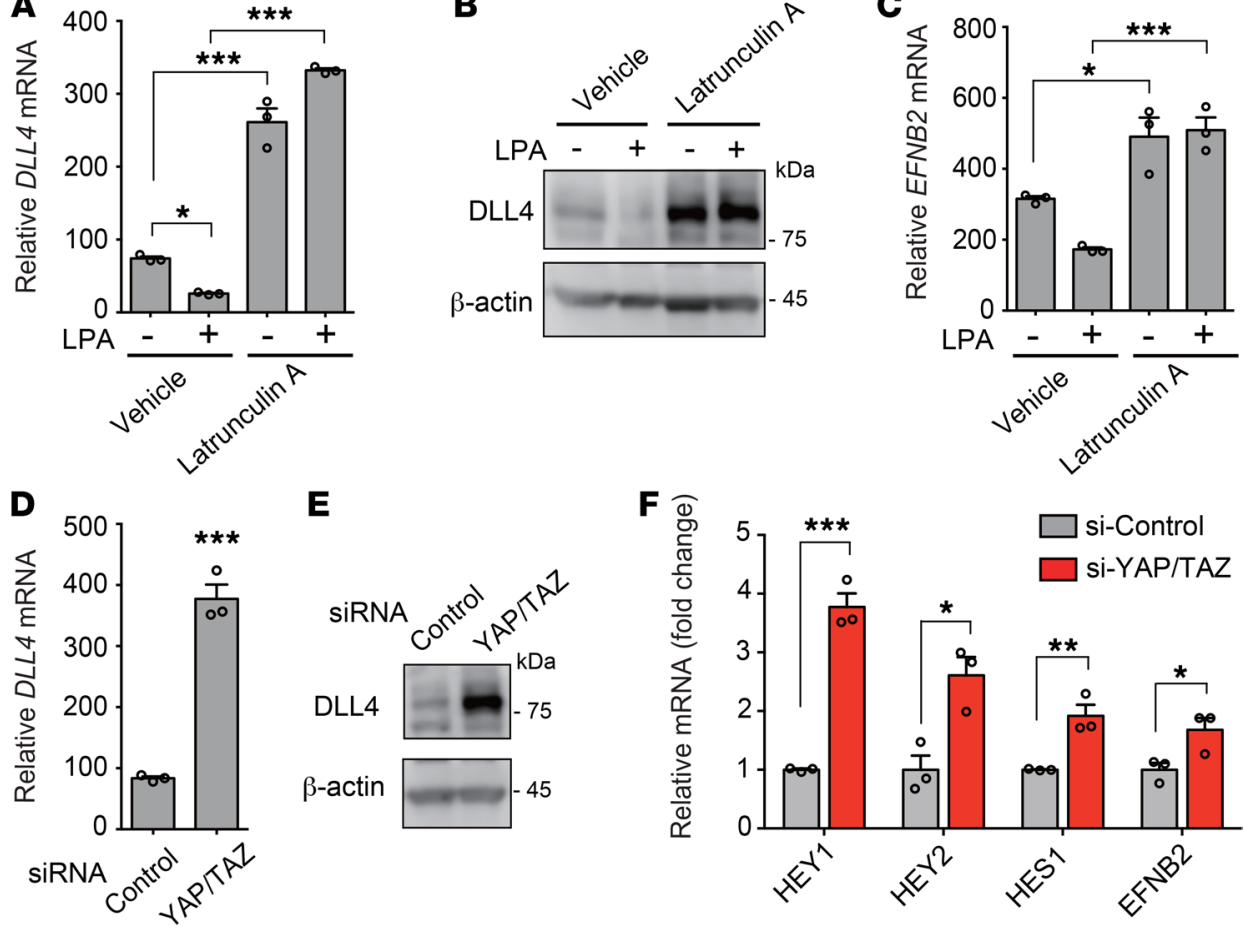

E
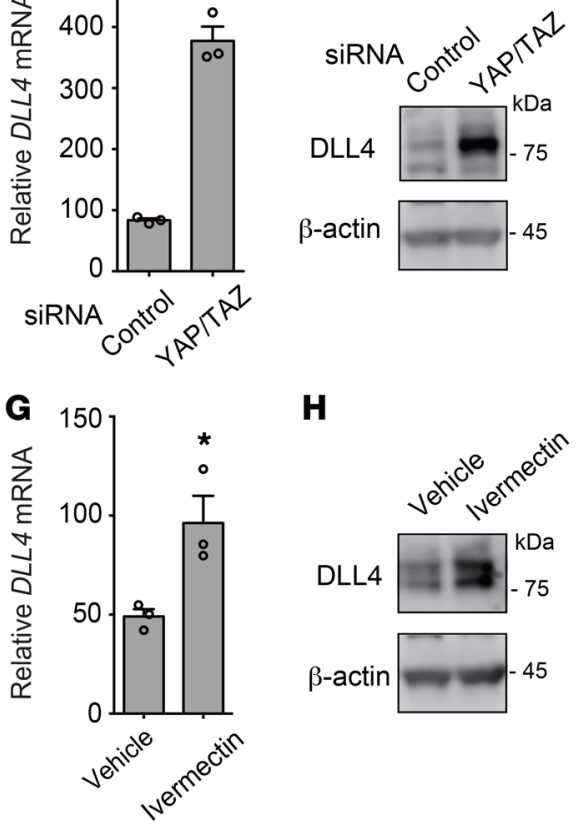

H
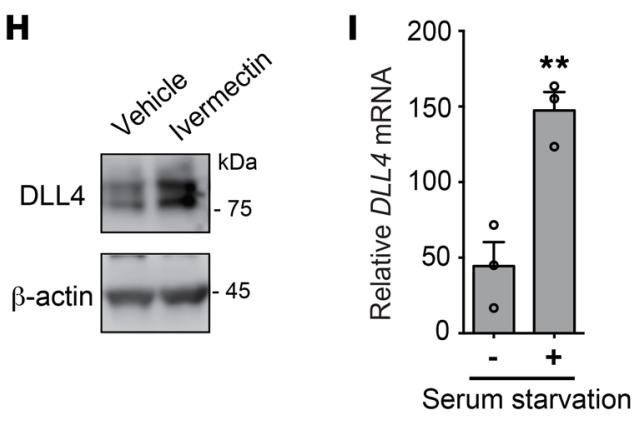

$\mathbf{J}$

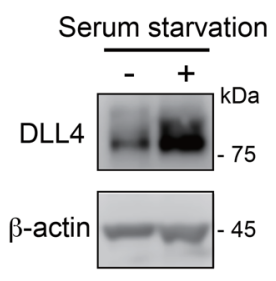

Figure 6. LPA-induced YAP/TAZ activation suppresses DLL4 expression in ECs. (A-C) Latrunculin $\mathrm{A}(1 \mu \mathrm{M}, 10$ minutes pretreatment) inhibited LPA-induced (LPA $10 \mu \mathrm{M}, 3$ hours) reduction of DLL4 mRNA (A), DLL4 protein (B), and EFNB2 mRNA (C) expression in serum-starved HUVECs. (D and E) YAP/TAZ siRNAs increased mRNA (D) and protein (E) expression of DLL4. (F) YAP/TAZ siRNAs increased mRNA expression of Notch target genes. (G and $\mathbf{H})$ Ivermectin $(10 \mu \mathrm{M}, 24$ hours treatment) increased mRNA (C) and protein (H) expression of DLL4. (I and J) A 12-hour serum starvation increased mRNA (I) and protein (J) expression of DLL4. ( $\mathbf{K}$ and $\mathbf{L}$ ) An overconfluent cell culture condition increased mRNA (K) and protein (L) expression of DLL4. HUVECs were serum-starved for 8 hours or transfected with siRNAs for 96 hours. Data are mean \pm SEM of triplicates. ${ }^{*} P<0.05,{ }^{* *} P<0.01$, ${ }^{* * *} P<0.001,1$-way ANOVA followed by Tukey's multiple-comparisons test ( $\mathbf{A}$ and C) or 2-tailed unpaired Student's $t$ test (D, $\mathbf{F}, \mathbf{G}, \mathbf{I}$, and $\mathbf{K})$. Unprocessed original scans of Western blots are shown in Supplemental Figure 13.

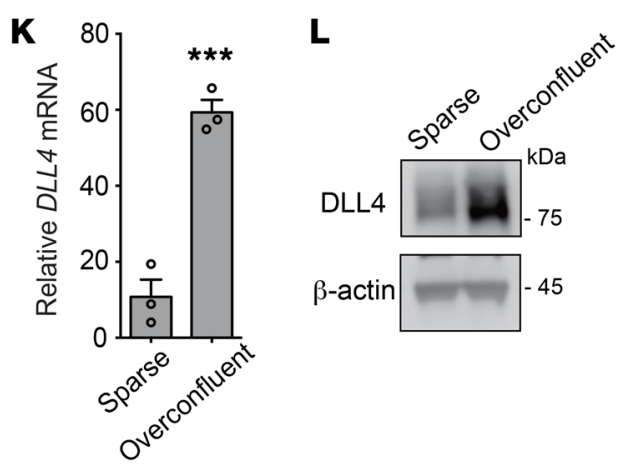

(Supplemental Figure 10B). Consistently, overexpression of the YAP-S94A mutant, which is unable to interact with TEAD (45), reduced DLL4 expression to the same degree as WT YAP in sparse HUVEC culture conditions (Figure 10, C and D). Overexpression of the YAP-5SA mutant, which mainly localized in the nucleus, suppressed the DLL4 expression in overconfluent culture conditions (Figure 10, E and F).

YAP/TAZ suppress $\beta$-catenin- and NICD-mediated DLL4 induction. DLL 4 transcription is promoted by Akt activation in HUVECs $(22,46)$. $\beta$-Catenin and NICD are indispensable for this mechanism. Therefore, we addressed whether YAP/TAZinduced DLL4 suppression involves these molecules in HUVECs. Interestingly, siRNA-mediated depletion of YAP/TAZ sensitized HUVECs to the angiogenic factors VEGF-A and Ang-1 and also to serum, all of which are known to activate Akt signaling (Figure 11, A-C). We found that the Akt inhibitor MK2206 considerably attenuated the upregulation of mRNA and protein expression of DLL4 caused by YAP/TAZ depletion (Figure 11, D and E) or Y27632 (Figure 11, F and G), while the basal DLL4 expression was marginally affected by the inhibitor in each treatment. 
A
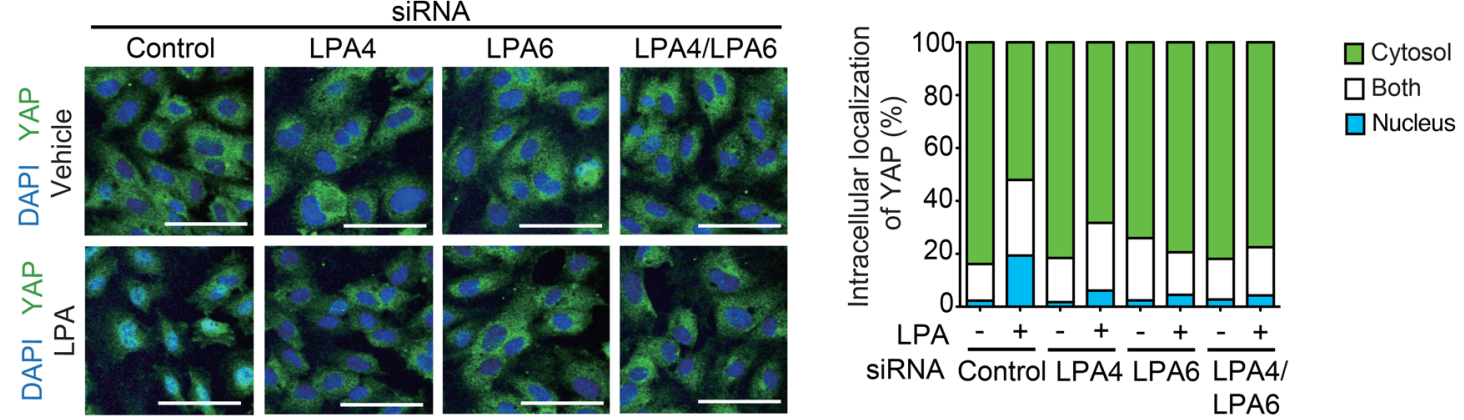

B

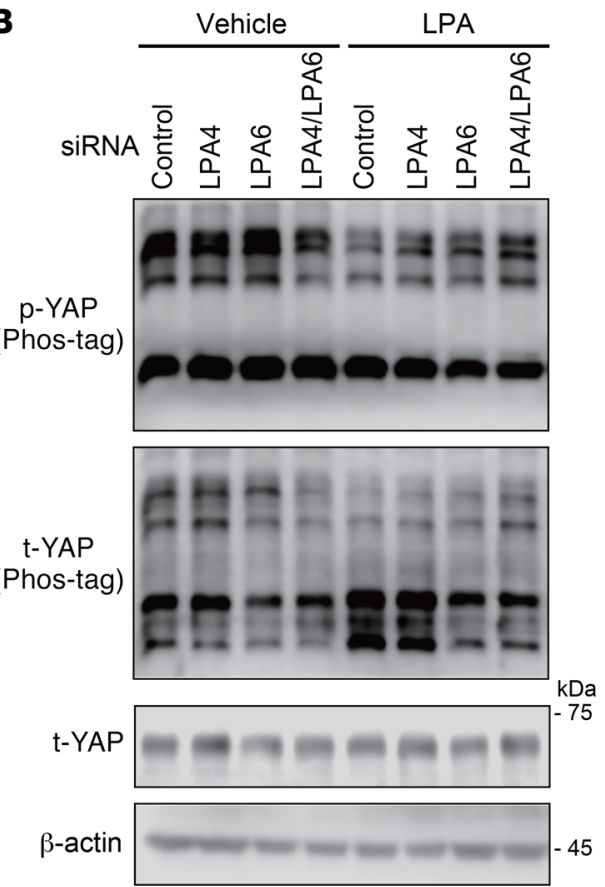

E
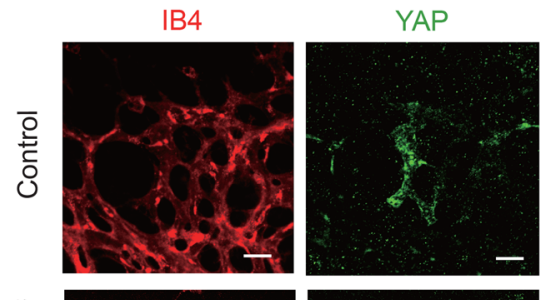

c
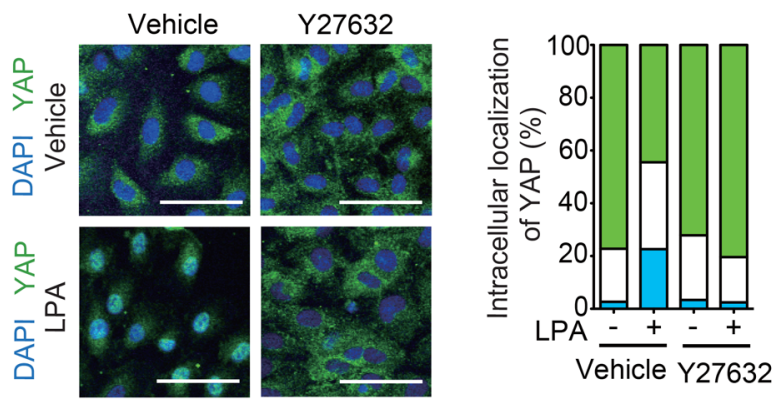

D
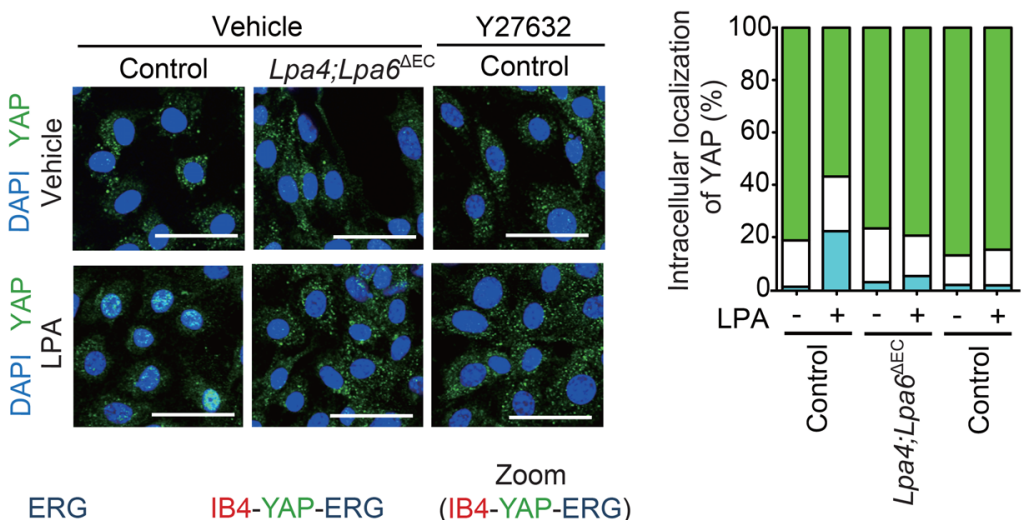

Zoom

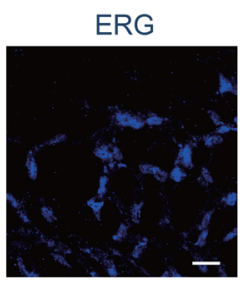

IB4-YAP-ERG

(IB4-YAP-ERG)
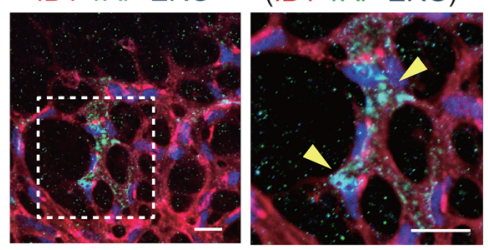

Vehicle $\overline{Y 27632}$
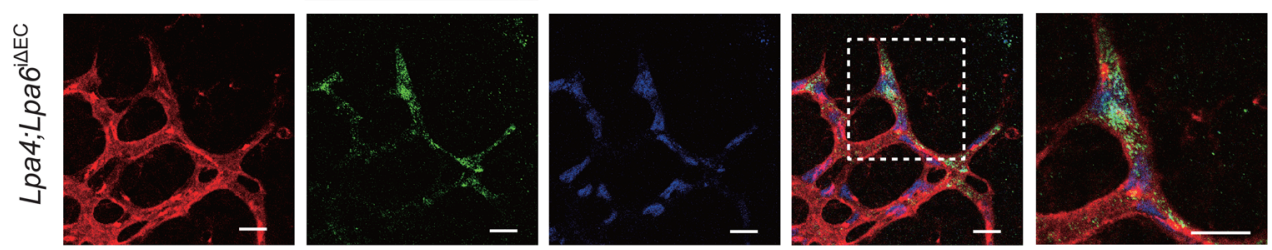

Figure 7. LPA4 and LPA6 are responsible for LPA-induced YAP activation in ECs. (A) LPA4/LPA6 siRNAs reduced LPA-induced (LPA $10 \mu M, 1$ hour) YAP nuclear localization in serum-starved HUVECs. Representative confocal images (left) and quantification data of YAP cellular localization (right; $n=293-394$ cells) are shown. Scale bars: $50 \mu \mathrm{m}$. (B) LPA4/LPA6 siRNAs reduced LPA-induced (LPA $10 \mu \mathrm{M}, 30$ minutes) YAP dephosphorylation in serum-starved HUVECs. Immunoblotting was performed with phospho-YAP (Ser127) and total YAP antibodies. YAP phosphorylation was assessed by differential migration on gels containing Phos-tag. Unprocessed original scans of Western blots are shown in Supplemental Figure 13. (C) Y27632 (10 $\mu \mathrm{M}, 10$ minutes pretreatment) reduced LPA-induced (LPA $10 \mu \mathrm{M}$, 1 hour) YAP nuclear localization in serum-starved HUVECs. Representative confocal images (left) and quantification data of YAP cellular localization (right; $n=$ 409-539 cells) are shown. Scale bars: $50 \mu \mathrm{m}$. (D) LPA4/LPA6 deficiency or Y27632 (10 $\mu \mathrm{M}, 10$ minutes pretreatment) reduced LPA-induced (LPA $10 \mu \mathrm{M}, 1$ hour) YAP nuclear localization in serum-starved mouse lung ECs. Representative confocal images (left) and quantification data of YAP cellular localization (right; $n=96-139$ cells) are shown. Scale bars: $50 \mu \mathrm{m}$. (E) Representative confocal images of YAP of P5 retinal vessels at the angiogenic front of $L p a 4$; $L p a 6^{i \Delta E C}$ and control littermates. Retinas were costained for isolectin B4 (IB4; red), YAP (green), and ERG (for EC nucleus; blue). Dashed boxes in the merged pictures indicate the areas for higher-magnification images shown in the far right panels. Yellow arrowheads indicate nuclear YAP. Scale bars: $20 \mu \mathrm{m}$. One retina was analyzed per mouse ( $n=4$ mice). 
A

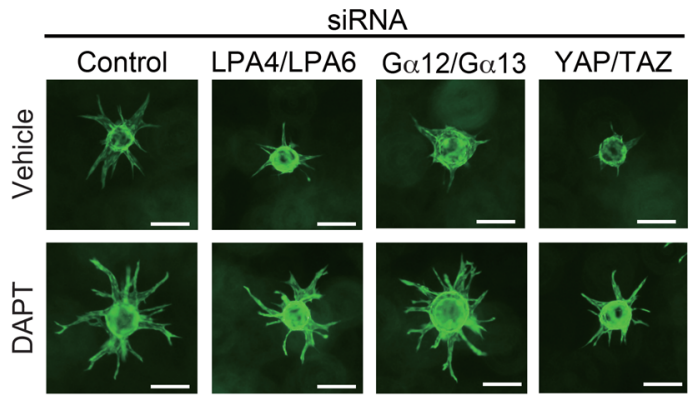

B

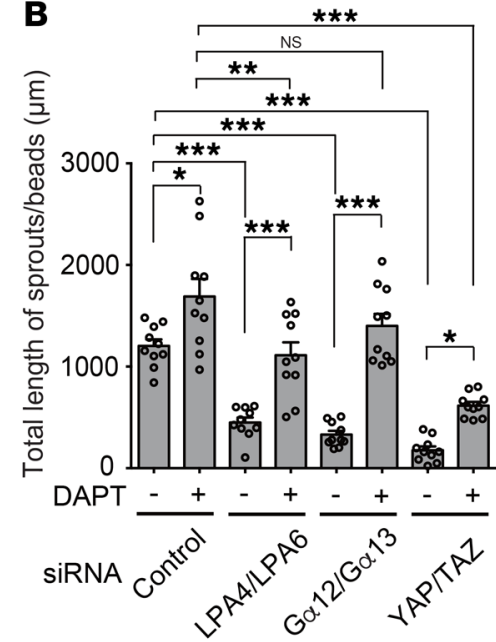

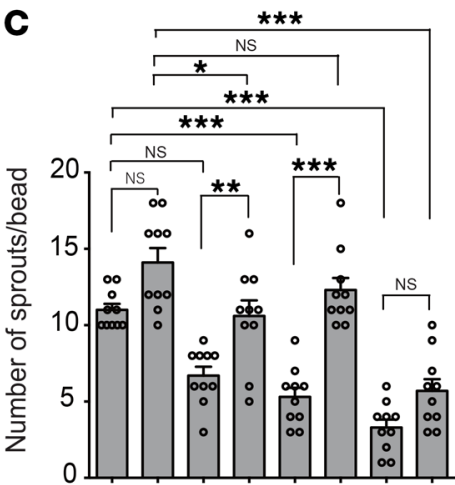

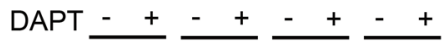
SiRNA

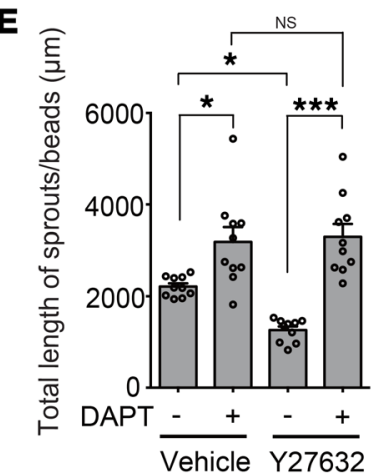

$\mathbf{F}$

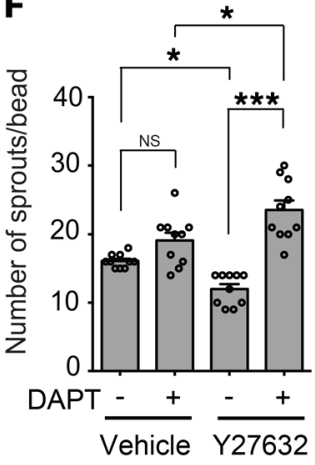

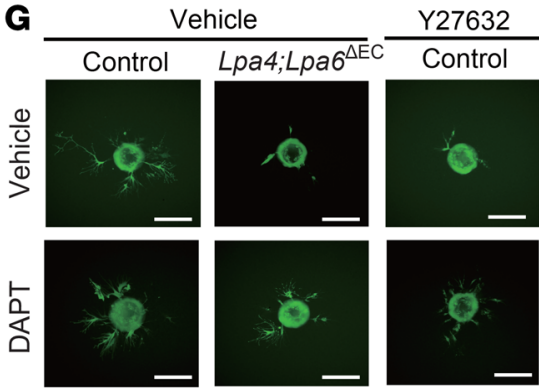

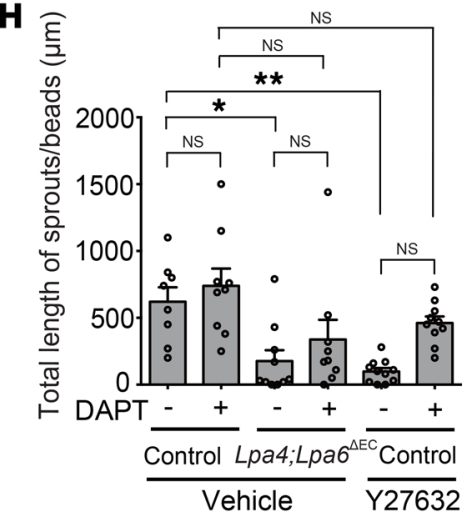

I

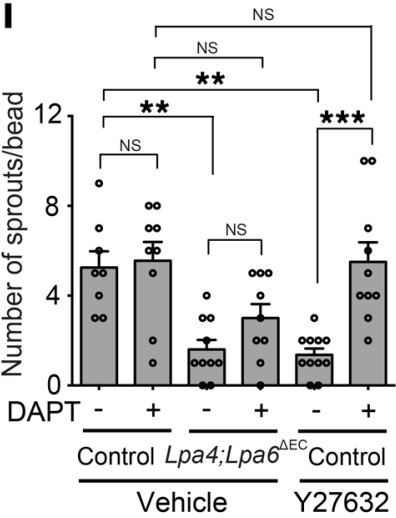

Figure 8. Impairment of EC sprouting by LPA4/LPA6-Ga12/G $\alpha 13-Y A P / T A Z$ signaling blockade is ameliorated by a Notch inhibitor. (A-C) LPA4/LPA6, $\mathrm{G} \alpha 12 / \mathrm{G} \alpha 13$, or YAP/TAZ siRNAs consistently suppressed the length and number of sprouts from the HUVEC-coated beads. DAPT ( $1 \mu \mathrm{M})$ significantly ameliorated the sprouting defects. Representative fluorescence images of sprouting are shown in A. Scale bars: $100 \mu \mathrm{m}$. Quantitative analyses of total length (B) and number $(\mathbf{C})$ of sprouts were performed. Data are mean \pm SEM $(n=10$ beads). (D-F) Y27632 $(10 \mu M)$ suppressed the length and number of sprouts from the HUVEC-coated beads. DAPT $(1 \mu \mathrm{M})$ significantly ameliorated the sprouting defects. Representative fluorescence images of sprouting are shown in $\mathbf{D}$. Scale bars: $100 \mu \mathrm{m}$. Quantitative analyses of length (E) and number (F) of sprouts were performed. Data are mean \pm SEM $(n=10$ beads). (G-I) LPA4/LPA6 deficiency or Y27632 $(10 \mu \mathrm{M})$ treatment suppressed the length and number of sprouts from mouse lung EC-coated beads. DAPT (10 $\mu \mathrm{M})$ significantly ameliorated the sprouting defects. Representative fluorescence images of sprouting are shown in G. Scale bars: $100 \mu \mathrm{m}$. Quantitative analyses of total length (H) and number (I) of sprouts were performed. Data are mean \pm SEM $\left(n=8-11\right.$ beads). ECs are stained green with calcein. ${ }^{*} P<0.05,{ }^{* *} P<0.01,{ }^{* * *} P<0.001$, 1-way ANOVA followed by Tukey's multiple-comparisons test.

In addition, the mRNA and protein induction of DLL4 by YAP/ TAZ depletion did not occur in the absence of $\beta$-catenin (Figure $11, \mathrm{H}$ and I). Consistently, $\beta$-catenin depletion greatly attenuated the mRNA induction of DLL4 and of Notch target genes that was caused by latrunculin A treatment (Supplemental Figure 11).
Similarly to $\beta$-catenin depletion, Notch inhibition by DAPT abolished the upregulation of mRNA and protein expression of DLL4 by YAP/TAZ siRNAs (Figure 11, D and E) and Y27632 (Figure 11, $\mathrm{F}$ and $\mathrm{G})$. These results suggest that endothelial YAP/TAZ attenuate $\beta$-catenin- and NICD-mediated DLL4 induction. 
A

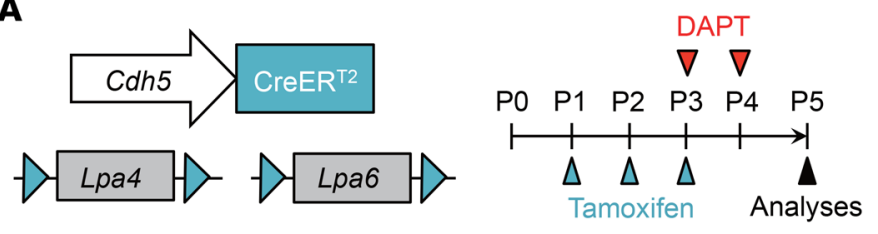

B

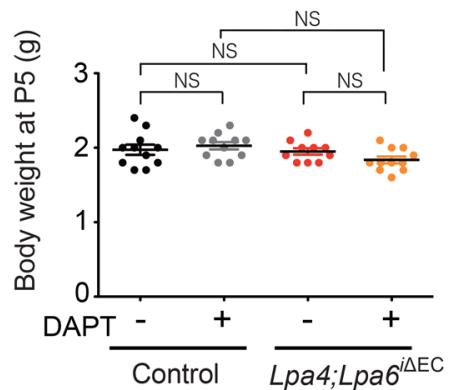

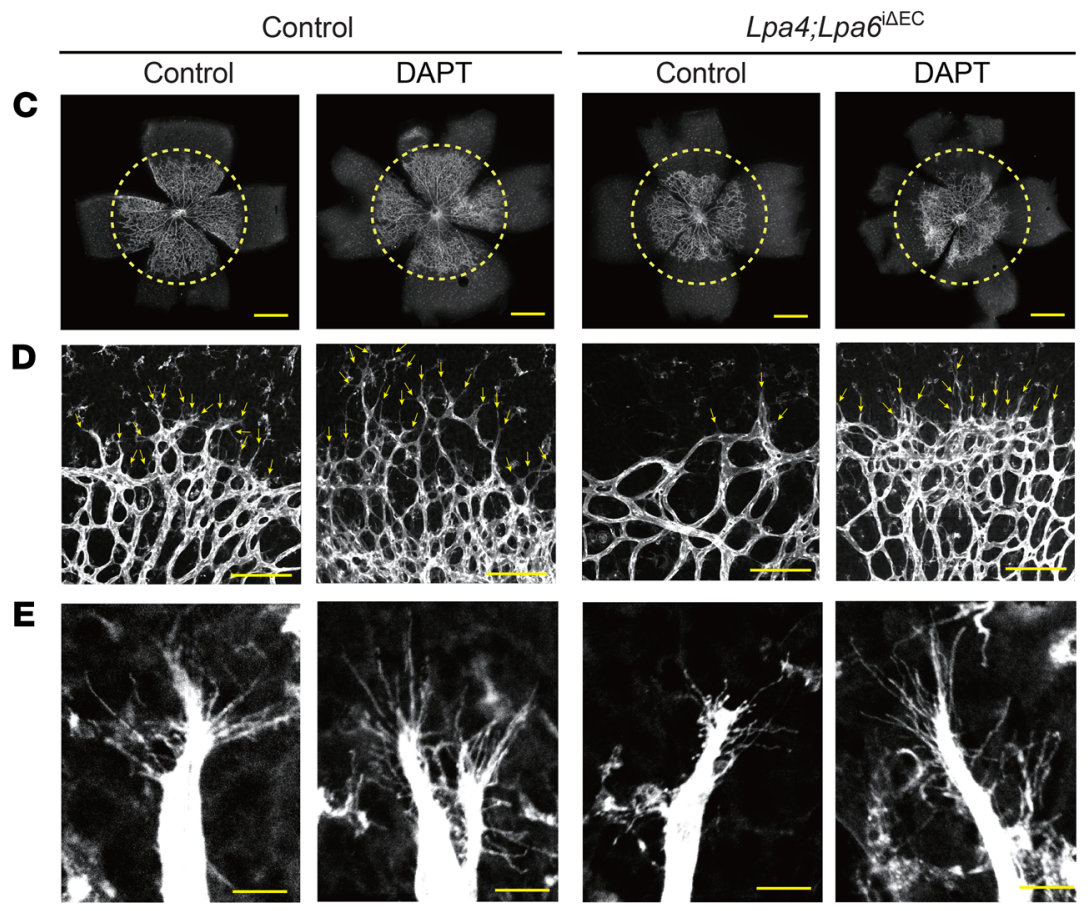

Figure 9. A Notch inhibitor ameliorates impaired retinal angiogenesis due to endothelial-specific Lpa4/ Lpa6 deletion. (A) $L p a 4 ; L p a 6^{i \Delta E C}$ and control littermates were injected with DAPT $(50 \mathrm{mg} / \mathrm{kg})$ at P3 and $\mathrm{P} 4$, and the retinas were prepared at P5. Vessels were stained with isolectin B4. (B) Body weight of DAPT-treated $L p a 4 ; L p a 6^{i \triangle E C}$ and control littermates at P5. (C) Representative confocal images of DAPT-treated $L p a 4 ; L p a G^{i L E C}$ and control retinas. Yellow dashed circles indicate the front edge of radial expansion of vehicle-treated control retina. Scale bars: $500 \mu \mathrm{m}$. (D) Representative confocal images of retinas at the edge of the angiogenic growth front. Sprouts are indicated by yellow arrows. Scale bars: $100 \mu \mathrm{m}$. (E) Representative high-magnification confocal images of retinas at the edge of the angiogenic growth front. Scale bars: $10 \mu \mathrm{m}$. (F-K) Quantitative analyses of retinal vessel expansion $(\mathbf{F})$, vascular area $(\mathbf{C})$, branch point number $(\mathbf{H})$, sprout number (I), filopodia number (J), and filopodia length (K). Each dot represents the value of a mouse. Horizontal lines represent mean \pm SEM $(n=10-11$ mice). One retina was analyzed per mouse. ${ }^{*} P<0.05$, ${ }^{*} P<0.01,{ }^{* *} P<0.001,1$-way ANOVA followed by Tukey's multiple-comparisons test.

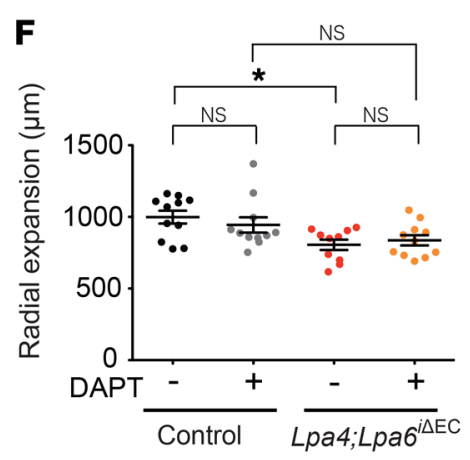

G

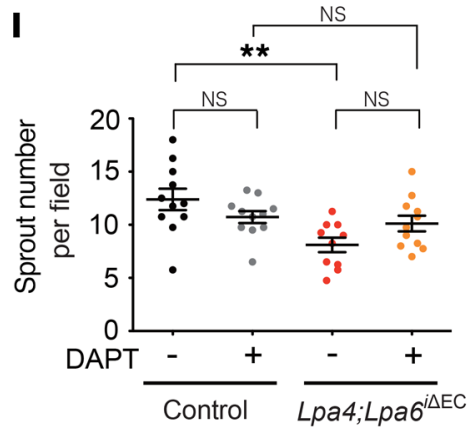

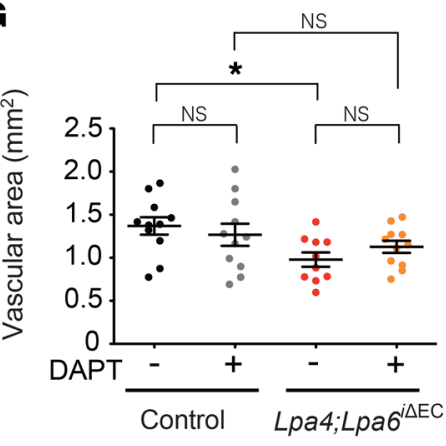

$\mathbf{J}$

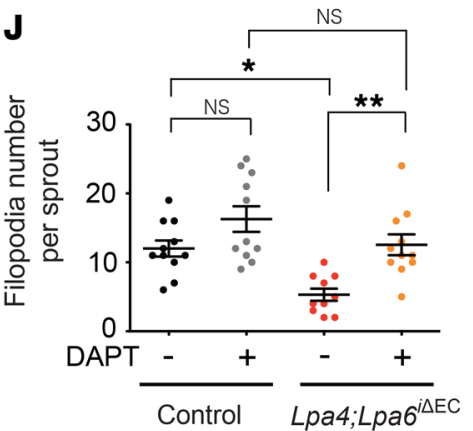

H

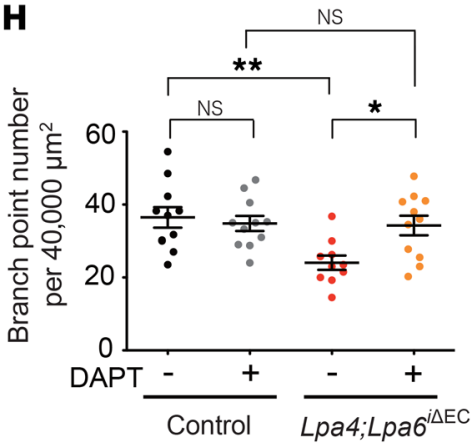

$\mathbf{K}$

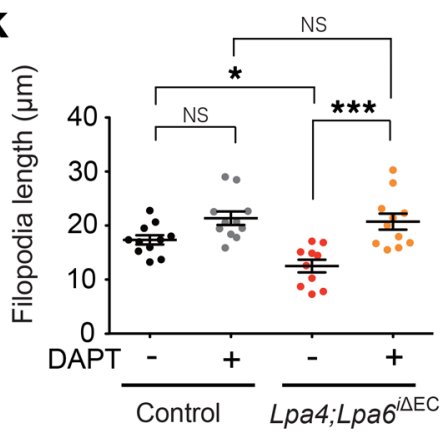



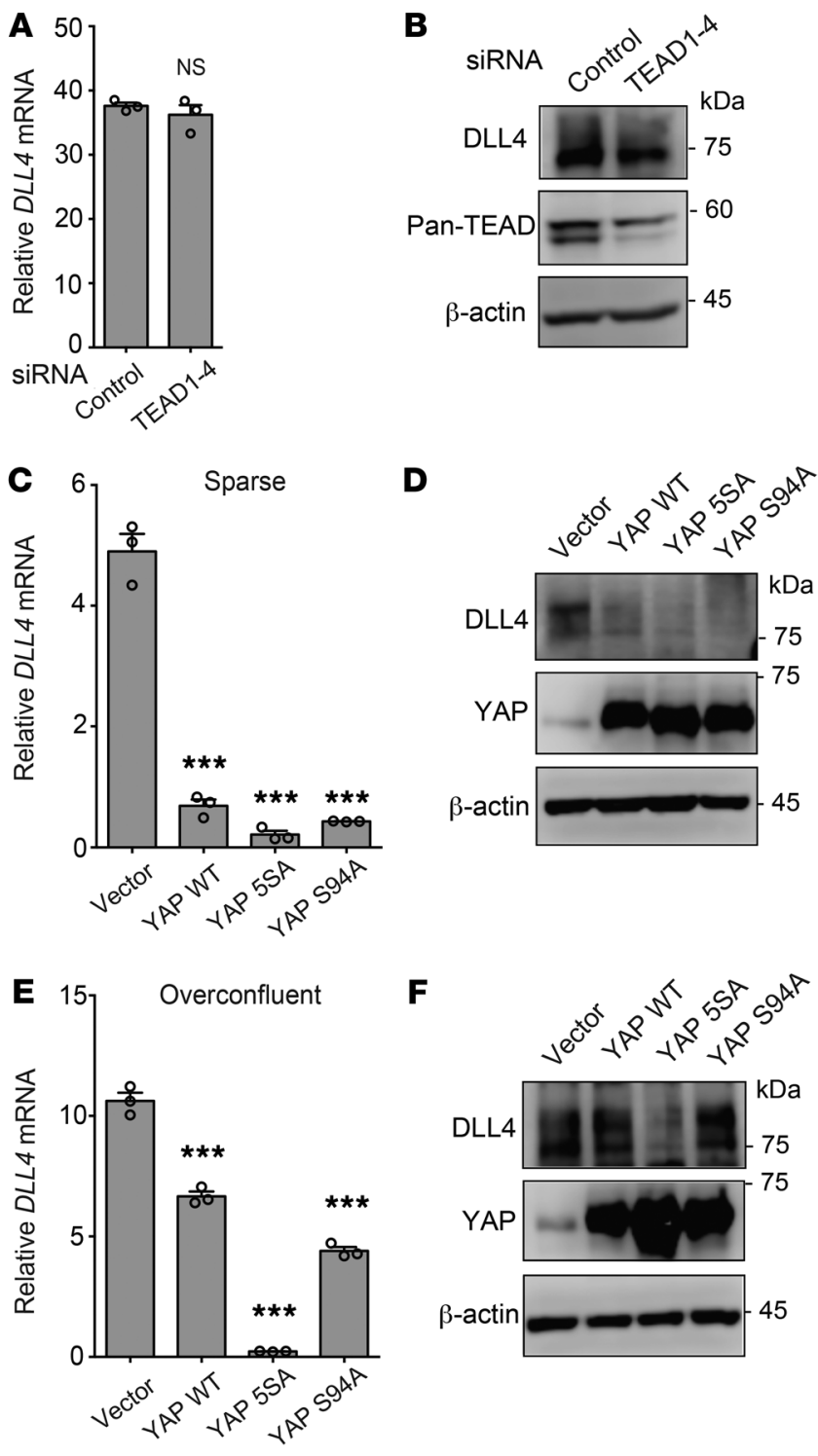

\section{Discussion}

In this study, we identified LPA4 and LPA6 as LPA receptors responsible for embryonic angiogenesis. All Lpa4;Lpa6-DKO embryos died by E11.5 due to global vascular deficiencies that were not seen in mice deficient in either Lpa 4 or Lpa6. We note that the vascular phenotypes of Lpa4;Lpa6-DKO embryos, such as pericardial effusion, enlarged dorsal aortae, and poor vascular networks, are very similar to those of Atx-KO, Gna12; Gna13-DKO, and Rock1;Rock2-DKO mice (12-14). These results

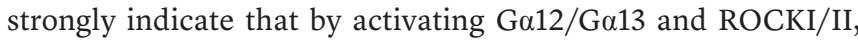
both LPA4 and LPA6 mediate LPA-induced embryonic angiogenesis in a coordinated manner.

Certain anomalies of Lpa4;Lpa6-DKO embryos, such as pericardial effusion, axial turning defects, and apparent lack of mesodermal segmentation, have been commonly observed in embryos with gain-of-function and loss-of-function mutations for Notch signaling $(25,27,47)$. Given that Dll4-overexpressing mice (as gain-of-function mutants; ref. 27) possessed enlarged aortae as observed in Lpa4;Lpa6-DKO mice, it is likely that LPA-LPA4/
Figure 10. Nuclear YAP suppresses DLL4 expression in a TEAD-independent manner. (A and B) TEAD1-4 siRNAs had no significant effect on mRNA (A) or protein (B) expression of DLL4. (C and D) Overexpression of YAP WT and 5SA and S94A mutants reduced mRNA (C) and protein (D) expression of DLL4 in sparse HUVECs. (E and F) Overexpression of the YAP 5SA mutant reduced mRNA (E) and protein (F) expression of DLL4 in overconfluent HUVECs. Data are mean \pm SEM of triplicates. ${ }^{* * *} P<0.001,1$-way ANOVA followed by Dunnett's multiple-comparisons test. Unprocessed original scans of Western blots are shown in Supplemental Figure 14.

LPA6 signaling suppresses Notch signaling during embryonic development. This conclusion is supported by the phenotypes of Notch1;Notch4-DKO and Dll4-Het embryos (as loss-of-function mutants; refs. 25, 47), both of which exhibited decreased diameter of the dorsal aortae.

Selective binding of ligand-activated GPCRs to their appropriate $\mathrm{G} \alpha$ proteins is critical for signal transduction (48). In this study, we used HUVECs, which endogenously expressed LPA1, $L P A 4$, and LPA6, to reveal the mechanisms behind LPA4/LPA6mediated angiogenesis. Luciferase reporter assay showed that LPA activates the Ga12/Ga13-Rho-ROCK signaling pathway, which was attenuated by LPA4 and/or LPA6 siRNAs, but not the LPA1/LPA3 antagonist Ki16425. On the other hand, our examinations of calcium influx and intracellular cAMP level changes indicated that LPA did not activate Gaq, Gai, or Gas protein in HUVECs. We previously reported that heterologously

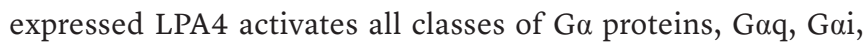
Gas, and $G \alpha 12 / G \alpha 13$ proteins, in some cells other than HUVECs (6). Thus, the coupling efficiency of LPA4 to Gaq, Gai, and Gas proteins might depend on the cell type, the culture conditions, or the receptor expression levels. With regard to LPA6, studies by 3 independent groups, including us, have established a con-

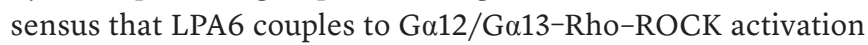
pathways $(8,49,50)$. Given that Ki16425 had no effect on the Ga12/Ga13-Rho-ROCK signaling pathway, LPA1 is unlikely to couple with $\mathrm{G} \alpha 12 / \mathrm{G} \alpha 13$ in HUVECs. Instead, LPA1 has been

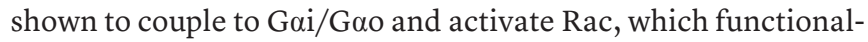
ly antagonizes Rho $(51)$, in primary culture cells $(52,53)$. However, we detected little or no LPA1 signal in HUVECs, probably as a result of the same coupling inefficiency as observed with LPA4. Taken together, our results suggest that endothelial LPA4/LPA6 signaling contributes to angiogenesis mainly by activation of Ga12/Ga13. This appears to be consistent with previous reports by Guan and colleagues that LPA and thrombin activate YAP/ TAZ through specific GPCRs that couple to $\mathrm{G} \alpha 12 / \mathrm{G} \alpha 13(30,54)$, as will be discussed later.

The question remains of why 2 LPA receptors are essential in ECs for proper angiogenesis. Previously, we revealed that LPA4 and LPA6 had distinct ligand selectivities for LPA species (6). For example, LPA6 is more potently activated by LPA with an acyl chain at the $s n$-2 position (2-acyl-LPA) rather than the $s n-1$ position (1-acyl-LPA), whereas LPA4 shows a ligand preference for 1-acyl-LPA over 2-acyl-LPA. Therefore, both LPA species appear to be sensed effectively by the coordinated action of endothelial LPA4 and LPA6, enabling them to contribute to proper angiogenesis. 


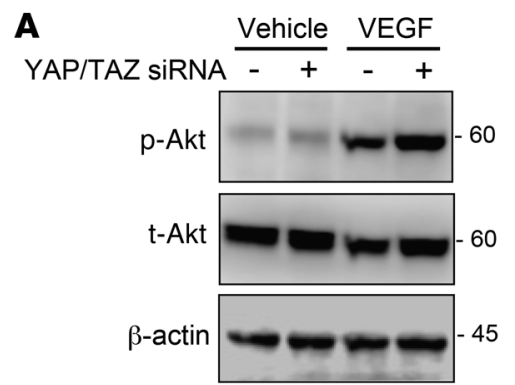

D

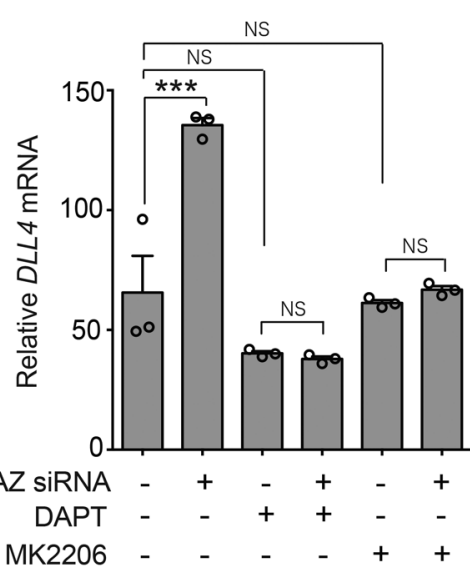

$\mathbf{F}$

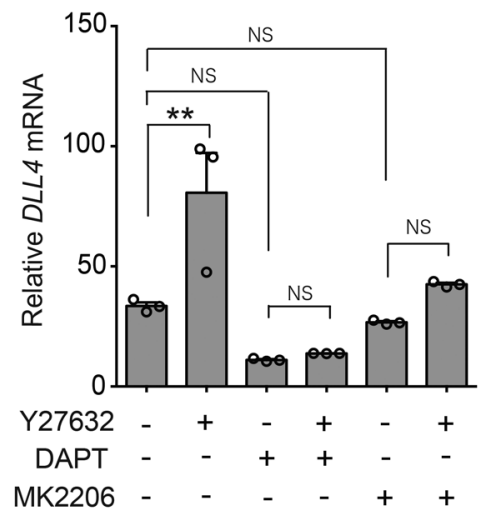

H

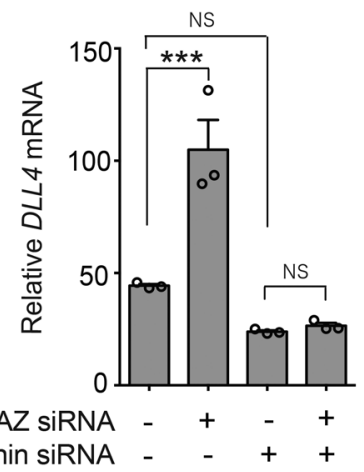

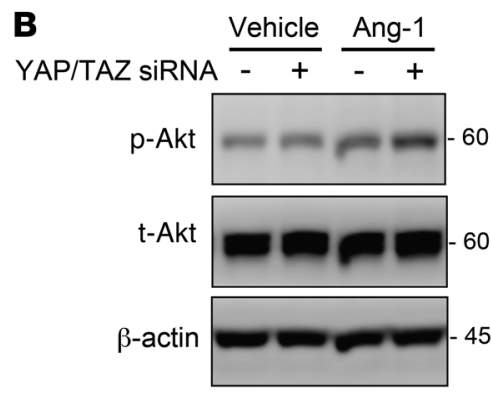

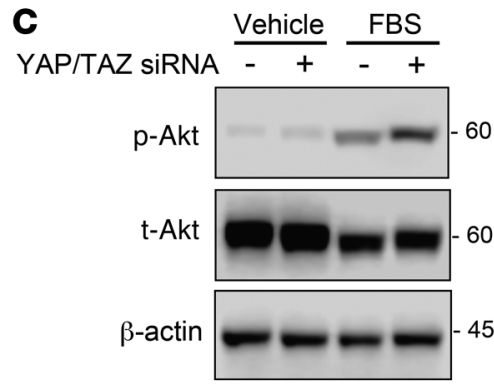

E

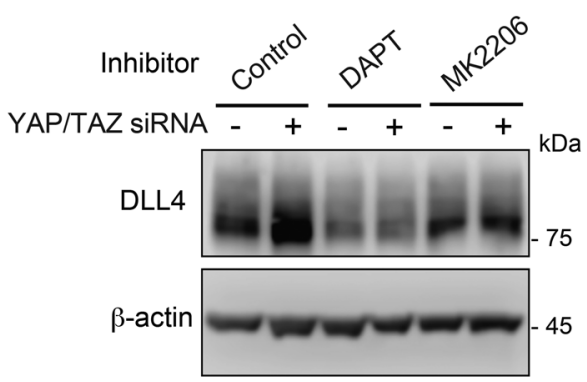

G
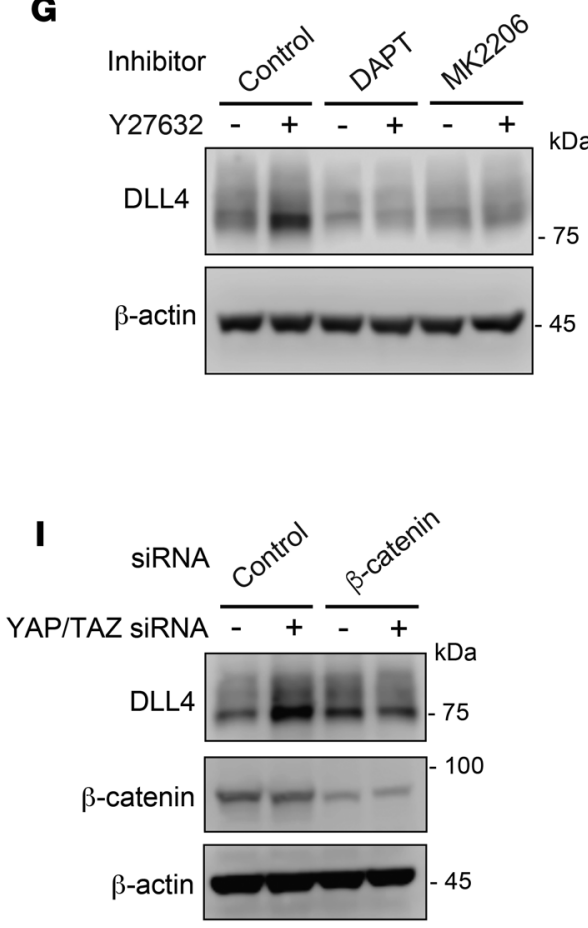

Figure 11. YAP/TAZ suppress $\beta$-catenin- and NICD-mediated DLL4 induction. (A-C) YAP/TAZ siRNA sensitized HUVECs to Akt activation in response to $50 \mathrm{ng} / \mathrm{mL}$ VEGF-A (A) and $400 \mathrm{ng} /$ $\mathrm{mL}$ Ang-1 (B), and 10\% FBS (C) for 10 minutes. ( $\mathbf{D}$ and $\mathbf{E})$ DAPT ( $10 \mu \mathrm{M}, 3$ hours) or MK2206 (10 $\mu \mathrm{M}, 3$ hours) abolished YAP/TAZ siRNA-induced mRNA (D) and protein (E) expression of DLL4. (F and C) DAPT $(10 \mu \mathrm{M}, 10$ minutes pretreatment) or MK2206 $(10 \mu \mathrm{M}, 10$ minutes pretreatment) attenuated mRNA (F) and protein (G) expression of DLL4 induced by Y27632 (10 $\mu \mathrm{M}, 3$ hours). (H and I) $\beta$-Catenin siRNA abolished YAP/TAZ siRNA-induced mRNA $(\mathbf{H})$ and protein (I) expression of DLL4. Data are mean \pm SEM of triplicates. ${ }^{* *} P<0.01,{ }^{* *} P<$ $0.001,1$-way ANOVA followed by Tukey's multiple-comparisons test. Unprocessed original scans of Western blots are shown in Supplemental Figure 15.
Some endothelial GPCRs, such as thrombin receptor (PAR1) and sphingosine-1-phosphate receptor (S1P1), thus far have been suggested to control developmental angiogenesis by the phenotypes of mice deficient in each receptor $(55,56)$. However, as far as we know, there have been no reports on GPCRs that mediate developmental angiogenesis by regulating DLL4/Notch signaling in ECs. Our in vitro studies using HUVECs demonstrated that LPA4 and LPA6 suppress DLL4 expression in a coordinated manner. Consistent with this, we revealed that concomitant loss of LPA4 and LPA6 in retinal ECs resulted in increased expression of Dll4, suggesting that endothelial LPA4/LPA6 signaling regulates Dll4 expression also in vivo.

We also demonstrated that pharmacological and siRNAmediated targeting of YAP/TAZ increased the expression of DLL4 and its target genes. Although a recent study reported similar 


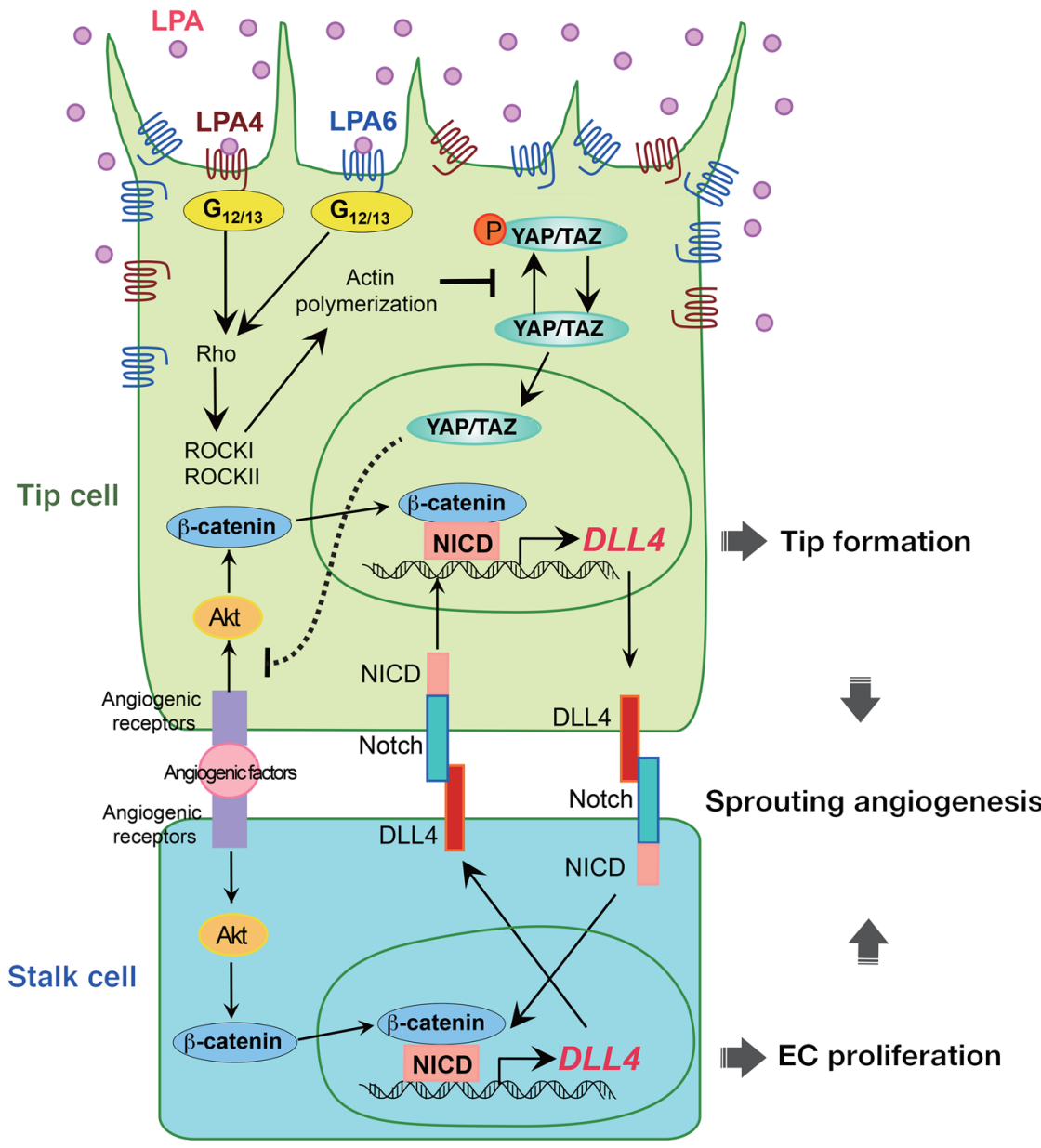

Figure 12. A working model showing that LPA regulates sprouting angiogenesis via a mechanism that involves LPA4/LPA6, G $\alpha 12 / G \alpha 13$, Rho, ROCK, actin polymerization, YAP/TAZ, and DLL4. Endothelial LPA4 and LPA6 activate G $\alpha 12 / G \alpha 13-R h o-R O C K$ signaling, which induces actin polymerization and nuclear localization of YAP/TAZ. Nuclear YAP/TAZ repress DLL4 expression by inhibiting the Akt signaling.

results in HUVECs (36), it is noteworthy that the Notch inhibitor DAPT restored the sprouting defects of YAP/TAZ siRNA-treated HUVECs in our study. YAP/TAZ-mediated DLL4 suppression is likely to be involved in LPA4/LPA6-mediated sprouting angiogenesis, as DAPT again restored sprouting defects in LPA4/LPA6 siRNA-treated HUVECs. More importantly, DAPT ameliorated impaired retinal angiogenesis in $L p a 4 ; L p a 6^{\text {IAEC }}$ mice as seen in Nrf2-KO, Jag1-KO, and Git1-KO mice (57-59). All of these mice displayed upregulated Dll4 expression in ECs. YAP nuclear localization was impaired in the angiogenic front of $L p a 4 ; L p a \sigma^{\text {iLEC }}$ retinas. Several independent studies have revealed that endothelial $\mathrm{YAP} / \mathrm{TAZ}$ mediate sprouting angiogenesis (31-36). In this context, we propose LPA4 and LPA6 as regulators of sprouting angiogenesis that repress DLL4 expression by activating YAP/TAZ in ECs.

Nuclear YAP in ECs plays a substantial role in sprouting angiogenesis in response to various stimuli $(31,32,34)$. In the present study, a reduction in both nuclear and cytoplasmic YAP by siRNA resulted in an increase in DLL4 expression in HUVECs. Meanwhile, when HUVECs were cultured under serum-starved or overconfluent conditions, nuclear localization of YAP was decreased concomitantly with an increase in DLL4 expression. Similar results were obtained by blocking of ROCK activity, which led to the predominant cytoplasmic retention of YAP. Furthermore, transient expression of the nuclear-localized YAP-5SA mutant remarkably reduced DLL4 expression. Thus, these results consistently suggest that nuclear YAP/ TAZ suppress endothelial DLL4 expression (Figure 12). Indeed, Barry et al. previously showed that transgenic overexpression of a phospho-deficient YAP mutant, which exhibits enhanced nuclear localization, repressed Dll4 gene expression in mouse intestinal epithelium (60). The involvement of nuclear YAP/TAZ in LPA4/LPA6-induced angiogenesis could provide a possible reason for the apparently milder phenotypes of Lpa4; $L p a 6^{i L E C}$ retinas compared with those observed in Yap;Taz ${ }^{i \perp E C}$ retinas $(31-33,36)$, as cytoplasmic YAP/TAZ also exert angiogenic functions in ECs. In particular, YAP/TAZ regulate CDC42 in the migration of tip ECs (33). A number of studies demonstrated that TEAD transcription factors mediate the nuclear function of YAP/TAZ (28). Although YAP/TAZ generally function as transcriptional coactivators, YAP/TAZ reportedly repress transcription of numerous target genes in the nucleus in association with TEAD $(44,61)$. Our present study, however, provides data supporting the idea that YAP/TAZ suppress DLL4 expression independently of TEAD.

In addition to the TEAD-independent function, our data suggested that YAP/TAZ repress Akt activity, which induces DLL4 expression in a manner dependent on $\beta$-catenin and NICD in ECs (Figure 12). This proposed molecular mechanism is plausible, given that NICD and $\beta$-catenin are indispensable for the Akt-induced DLL4 transcription in $\operatorname{HUVECs~}(22,46)$. Although Manderfield et al. demonstrated that YAP and NICD physically interact and modulate gene transcription in mouse aortic smooth muscle cells (62), we observed no obvious binding of YAP with NICD or with $\beta$-catenin in HUVECs under our experimental conditions (our unpublished observations). We therefore concluded that YAP/TAZ appear to repress DLL4 induction by inhibiting the Akt signaling pathway rather than by interacting directly with and inhibiting the function of NICD or $\beta$-catenin. Further studies, however, are warranted to clarify the detailed molecular mechanism. In this context, it is interesting to note that the stress-responsive transcription factor NRF2 represses endothelial DLL4 expression by blocking Akt signaling to promote sprouting angiogenesis (57).

Lipid phosphate phosphatase 3 (LPP3) is an ectoenzyme expressed on the surface of ECs and seems to contribute predominantly to the degradation of LPA in blood (63). Yukiura et al. reported that LPP3 is expressed locally in HUVECs, i.e., LPP3 is localized 
to cell-cell contact sites rather than noncontact sites (64). This report suggested that LPP3 is likely to restrict LPA signaling at the subcellular level and that endothelial LPA4/LPA6 signaling may be dominant in the angiogenic front of the mouse retinal vessels. This assumption is further in line with our observation that YAP localized preferentially in nuclei of mouse ECs at the angiogenic front in an LPA4/LPA6-dependent manner. The positional restriction of LPA4/LPA6 functions in retinal vessels may also account for the apparently milder phenotypes of $L p a 4 ; L p a 6^{i \triangle E C}$ retinas compared with the phenotypes of Yap; $\operatorname{Taz}^{i \triangle E C}$ retinas $(31-33,36)$, as endothelial YAP/TAZ are regulated by various extracellular cues other than LPA, including mechanical stimuli $(34,65)$ and receptor ligands $(32,54)$.

In cooperation with VEGF signaling, Notch signaling contributes to specify and balance the tip and stalk cell phenotypes during the angiogenic sprouting process (66). High expression of DLL4 in filopodia-rich endothelial tip cells, which lead and guide new sprouts (67), is thought to activate Notch and to suppress the tip cell phenotype in adjacent (stalk) ECs by reducing sensitivity to the angiogenic activity of VEGF-A. Additionally, Notch activation by Dll4-presenting tip cells has been suggested to act as a positive regulator of Dll4 transcription in adjacent stalk cells (Figure 12) (68). Notably, studies using mice deficient in Notch signaling inhibitor molecules, including Jagged1 and GIT1, revealed that stalk cells possess an intrinsic ability to reciprocally activate Notch in adjacent tip cells $(58,59)$. Jagged1 and GIT1 deficiencies remarkably enhanced D1l4 expression in both tip and stalk cells because of an amplified Dll4-Notch-Dll4 positive-feedback loop at the angiogenic front. Because LPA4/LPA6 signaling inhibits Notch signaling by repressing Dll4 transcription, the Dll4-NotchDll4 positive-feedback loop is probably amplified in ECs at the angiogenic front of $L p a 4 ; L p a 6^{i \triangle E C}$ retinas. Thus, we interpret that, as in Jag1-KO and Git1-KO ECs, Dll4 upregulation in Lpa4; $L p a 6^{i \triangle E C}$ ECs inhibits formation and also potentiation of tip cells. Indeed, we found that tip cells in the Lpa4;Lpa6 ${ }^{i \triangle E C}$ retina were less numerous and exhibited reductions in number and length of sprouting filopodia.

Sprouting angiogenesis is a multistep process including tip cell formation and stalk cell proliferation (66). In our in vitro assays, knockdown of LPA4/LPA6, Ga12/Ga13, or YAP/TAZ consistently led to sprouting defects of HUVECs. Furthermore, knockdown of LPA4/LPA6 blocked EC proliferation, suggesting that the LPA4/ LPA6-Ga12/Ga13-YAP/TAZ signaling pathway is involved in sprouting angiogenesis also by promoting the proliferation of EC. This is in agreement with previous reports that demonstrated that YAP and TAZ were required for EC proliferation $(31,33)$. Similarly, there is a report indicating that LPA promoted the proliferation of human corneal ECs via YAP activation (69).

In conclusion, we identified endothelial LPA4 and LPA6 as crucial receptors of LPA during sprouting angiogenesis. The LPA4/ LPA6-mediated Ga12/Ga13 signaling pathway likely downregulates DLL4 expression via YAP/TAZ activation (Figure 12). Sprouting angiogenesis is followed by vessel remodeling and pruning to form vascular networks under both developmental and pathological conditions. Thus, our findings may provide a molecular basis for targeting vascular development as a potential therapeutic strategy for pathological angiogenesis, particularly associated with cancer, age-related macular degeneration, rheumatoid arthritis, and psoriasis.

\section{Methods}

Further information can be found in Supplemental Methods.

Mice. Lpa4-KO mice were generated as previously described (16). Lpa4-floxed mice were generated by a modified CRISPR/Cas9 gene editing system (70). This system enables homologous recombination-mediated targeted loxP sequence integrations by using left and right single-guide RNA/Cas9 expression vectors and another vector with long double-stranded donor DNA spanning both left and right CRISPR target sequences (Supplemental Figure 2A). Briefly, we used the pX330 vector, provided by Feng Zhang (Addgene plasmid 42230) (71), to generate the single-guide RNA/Cas9 expression vectors. The left guide RNA sequence (5'-GAGAAAAGTCGATATTGAGC-3') and the right guide RNA sequence (5'-TGGAGGGTAAAAAAGCCATG-3') were inserted into the entry site of the pX330 vector, as reported by Mizuno et al. (72). These vectors were designated as pX330-Lpa4left and pX330-Lpa4-right, respectively. The donor vector, named pflox-Lpa4-dsDonor, was based on the pflox vector backbone, provided by Fumihiro Sugiyama (Supplemental Table 7) and designed to flank the exon containing the entire open reading frame of Lpa4 with loxP sites to allow for conditional removal by Cre recombinase. pflox-Lpa4-dsDonor contained a left homology arm (1249 bp) and a right homology arm (1755 bp) that flanked the left and right CRISPR target sequences, respectively. For microinjection, these $3 \mathrm{vec}-$ tors were isolated with a FastGene Gel/PCR Extraction Kit (Nippon Genetics, Tokyo, Japan) and diluted to $5 \mathrm{ng} / \mu \mathrm{L}$ (pX330-Lpa4-left/ right vectors) or $10 \mathrm{ng} / \mu \mathrm{L}$ (pflox-Lpa4-dsDonor) by deionized distilled water. After filtration with a $0.22-\mu \mathrm{m}$ filter (Millex-GV; Merck Millipore, Billerica, Massachusetts, USA), these vectors were microinjected into the male pronuclei of fertilized oocytes according to the standard protocols (73). Surviving 1-cell embryos were transferred into the oviducts of pseudopregnant ICR females. Lpa5-KO, Lpa6KO, and Lpa6-floxed mice (accession no. CDB0977K; http://www2. clst.riken.jp/arg/mutant\%20mice\%20list.html) were generated as previously described $(15,74)$. Tie2-Cre transgenic mice (75) were provided by RIKEN BioResource Research Center. Cdh5(PAC)-CreER $R^{T 2}$ transgenic mice were a gift from Ralf $\mathrm{H}$. Adams (37). All mice were on a C57BL/6 genetic background.

Analysis of retinal angiogenesis. To induce Cre-mediated recombination, $100 \mu \mathrm{g}$ of tamoxifen (Sigma-Aldrich) dissolved in ethanol/ corn oil $(1: 9, \mathrm{vol} / \mathrm{vol})$ at $40 \mathrm{mg} / \mathrm{mL}$ was administered to pups by daily oral gavage at P1 through P3. Eyes were retrieved from both male and female pups at P5 after euthanasia and fixed with $4 \%$ paraformaldehyde in PBS for 2 hours at $4^{\circ} \mathrm{C}$. After washing with PBS 4 times, retinas were isolated from the fixed eyes, and 4 radial incisions were performed to obtain 4 evenly sized quadrants. The retinas were then permeabilized and blocked in PBS containing 1\% BSA (Sigma-Aldrich) and $0.3 \%$ Triton $\mathrm{X}-100$ at room temperature for 1 hour. Blood vessels were stained with $10 \mu \mathrm{g} / \mathrm{mL}$ Alexa Fluor 594conjugated isolectin $\mathrm{B} 4$ (Thermo Fisher Scientific) overnight at $4^{\circ} \mathrm{C}$ in Pblec buffer $\left(1 \mathrm{mM} \mathrm{CaCl}_{2}, 1 \mathrm{mM} \mathrm{MgCl}_{2}, 0.1 \mathrm{mM} \mathrm{MnCl}_{2}\right.$, and $0.3 \%$ Triton X-100 in PBS). After washing with PBS 4 times, flat-mounted retinas were imaged using a laser scanning confocal microscope (LSM 780 META; Carl Zeiss). Analyses of radial expansion, vascular area, branch point number, and sprout number were conducted using ImageJ software (NIH). Briefly, radial expansion was measured as the distance of vessel growth from the optic nerve to the peripheral angiogenic front in each quadrant of the retina and averaged. 
The angiogenic front was defined as the line connecting the base of the sprouting ECs. Vascular area corresponded to the vascularized area divided by the field of view $(200 \mu \mathrm{m} \times 200 \mu \mathrm{m})$ in each quadrant. The average of each quadrant was calculated. Vascular area was measured by thresholding isolectin B4 signal. Threshold values were maintained constant throughout all measurements within each independent experiment. The number of vessel branch points was counted manually in a $200 \mu \mathrm{m} \times 200 \mu \mathrm{m}$ field located between an artery and a vein in each quadrant and averaged. The number of sprouts was counted manually in a $450-\mu \mathrm{m}$ length of the angiogenic front edge in each quadrant and averaged. For the analysis of filopodia, high-resolution confocal images were used. The number and length of filopodia per single sprout were measured. To inhibit Notch signaling, newborn pups were injected s.c. at P3 and P4 with $50 \mathrm{mg} / \mathrm{kg}$ DAPT (Tokyo Chemical Industry) dissolved in ethanol/ corn oil (1:9, vol/vol) at $5 \mathrm{mg} / \mathrm{mL}$. Control mice were injected with vehicle only. For staining of DLL4 or YAP, isolectin B4-stained retinas were washed 3 times for 10 minutes with $\mathrm{PBS}$ and then incubated overnight at $4^{\circ} \mathrm{C}$ in Pblec buffer with goat anti-DLL4 (diluted 1:40; AF1389; R\&D Systems) or rabbit anti-YAP antibody (diluted 1:100; 14074; Cell Signaling Technology). These primary antibodies were detected with appropriate Alexa Fluor 488-conjugated secondary antibodies by incubation at room temperature for 2 hours. Endothelial nuclei were stained with Alexa Fluor 647conjugated rabbit anti-ERG antibody (diluted 1:100; ab196149; Abcam). Total DLL4 staining intensities of a $300 \mu \mathrm{m} \times 600 \mu \mathrm{m}$ field at the angiogenic front and a 100- $\mu \mathrm{m}$ length of the artery were measured. Each intensity value was normalized to isolectin B4 area. Investigators were blinded to the genotype and treatment of animals during data analysis.

Fibrin gel sprouting assay. Fibrin gel sprouting assay was performed as described previously with some modifications (42). Briefly, HUVECs or mouse lung ECs were trypsinized and mixed with Cytodex 3 microcarrier beads (Sigma-Aldrich) at a ratio of $1 \times 10^{6}$ cells per 500 beads. The beads were coated with ECs by incubation for 4 hours at $37^{\circ} \mathrm{C}$ in a $15-\mathrm{mL}$ conical Falcon tube, with gentle shaking by hand every 20 minutes. The coated beads were then transferred to $3 \mathrm{~mL}$ of EGM-2 BulletKit containing 2\% FBS in a $35-\mathrm{mm}$ nonadherent dish. After 24 hours, the beads were washed with EGM-2 BulletKit 3 times and suspended in EGM-2 BulletKit containing 2\% FBS, $2 \mathrm{mg} / \mathrm{mL}$ fibrinogen (Sigma-Aldrich), $0.15 \mathrm{U} / \mathrm{mL}$ aprotinin (SigmaAldrich), and $10 \mathrm{ng} / \mathrm{mL}$ VEGF-A (Invitrogen). To inhibit Notch signaling in HUVECs or mouse lung ECs, 1 or $10 \mu \mathrm{M}$ DAPT $(10 \mathrm{mM}$ stock in DMSO) was further added, respectively. To form fibrin gel, 0.7-mL aliquots of the suspension were added to $4.5 \mu \mathrm{L}$ of $100 \mathrm{U} /$ $\mathrm{mL}$ thrombin in a 24-well plate. The plate was left for 5 minutes at room temperature and subsequently for 15 minutes at $37^{\circ} \mathrm{C}$. Mouse mesenchymal C3H10T1/2 cells (CCL-226; ATCC) were suspended at $2 \times 10^{4}$ cells $/ \mathrm{mL}$ in EGM-2 BulletKit supplemented with $2 \%$ FBS and $10 \mathrm{ng} / \mathrm{mL}$ VEGF-A and overlaid onto the fibrin gel layers at $1 \mathrm{~mL} /$ well. C3H1OT1/2 cells are considered to provide essential nutrients and growth factors to ECs, thus enabling them to mimic the stromal
cell-EC interaction in vitro (76). Subsequently, the cocultures were incubated at $37^{\circ} \mathrm{C}$ in $5 \% \mathrm{CO}_{2}$ for 96 or 120 hours. After removal of the adherent C3H10T1/2 cells by trypsinization, EC sprouts were labeled with $4 \mu \mathrm{g} / \mathrm{mL}$ calcein-AM (Dojindo) for 1 hour and photographed with a fluorescence microscope (IX 71; Olympus). The number and total length of sprouts were measured by ImageJ. The mean number of sprouts per bead was determined by counting of the sprouts originating directly from the cells lining the surface of the bead. The mean total length per bead represented the cumulative length of all sprouts per bead. For both parameters, 7-15 beads were assessed.

Statistics. All data are presented as the mean \pm SEM and were analyzed using GraphPad Prism 6 software (GraphPad Software). To calculate statistical significance between 2 groups, 2-tailed unpaired Student's $t$ test (for parametric analysis) or Mann-Whitney $U$ test (for nonparametric analysis) was performed. One-way ANOVA followed by Tukey's or Dunnett's multiple-comparisons test was used for comparisons among 3 or more groups. Two-way repeated-measures ANOVA followed by Bonferroni's multiple-comparisons test was used for the proliferation assay. Differences were considered statistically significant at $P$ less than 0.05 . No sample outliers were excluded. Individual in vitro experiments were performed at least 2 times with similar results. For in vivo experiments, data were collected from multiple independent experiments performed on different days with different mice.

Study approval. The animal experimental procedures used in this study were approved by the Institutional Animal Care and Use Committee of Akita University, Akita, Japan.

\section{Author contributions}

DY and SI designed the experiments and wrote the manuscript. DY, DK, and NA performed experiments and analyzed the data. TON supported retinal angiogenesis analysis. SM and ST established the Lpa4-floxed mouse line. YT and KY provided the Cdh5(PAC)-CreER ${ }^{T 2}$ mouse line and contributed to scientific discussion. All authors reviewed and approved the manuscript.

\section{Acknowledgments}

We thank T. Maruyama (Akita University), H. Igarashi (Akita University), Y. Kubota (Keio University), N. Takakura (Osaka University), H. Naito (Osaka University), and K. Yanagida (Harvard University) for their technical advice and useful discussions. We also thank K. Yasuda, K. Shigetsune, S. Takeuchi, and A. Sekiba (Akita University) for their technical support and Ralf $\mathrm{H}$. Adams (Cancer Research UK) for sharing the Cdh5(PAC)-CreER ${ }^{T 2}$ mouse line. This work was supported in part by Japan Society for the Promotion of Science KAKENHI grants JP16H05136, JP23116502, and JP25116703 (to SI) and JP16K08575 and JP26860182 (to DY) and grant from the Association for Research on Lactic Acid Bacteria (to SI).

Address correspondence to: Satoshi Ishii, Department of Immunology, Akita University Graduate School of Medicine, 1-1-1 Hondo, Akita-city, Akita 010-8543, Japan. Phone: 81.18.884.6089; Email: satishii@med.akita-u.ac.jp.
1. Tokumura A. A family of phospholipid autacoids: occurrence, metabolism and bioactions. Prog Lipid Res. 1995;34(2):151-184.

2. van Meeteren LA, Moolenaar WH. Regulation and biological activities of the autotaxin-LPA axis. Prog Lipid Res. 2007;46(2):145-160.

3. Aoki J. Mechanisms of lysophosphatidic acid production. Semin Cell Dev Biol. 2004;15(5):477-489.
4. Chun J, Hla T, Lynch KR, Spiegel S, Moolenaar WH. International Union of Basic and Clinical Pharmacology. LXXVIII. Lysophospholipid receptor nomenclature. Pharmacol Rev. 
2010;62(4):579-587

5. Shimizu T. Lipid mediators in health and disease: enzymes and receptors as therapeutic targets for the regulation of immunity and inflammation. Annu Rev Pharmacol Toxicol. 2009;49:123-150.

6. Yanagida K, Kurikawa Y, Shimizu T, Ishii S. Current progress in non-Edg family LPA receptor research. Biochim Biophys Acta. 2013;1831(1):33-41.

7. Noguchi K, Ishii S, Shimizu T. Identification of $\mathrm{p} 2 \mathrm{y} 9 / \mathrm{GPR} 23$ as a novel $\mathrm{G}$ protein-coupled receptor for lysophosphatidic acid, structurally distant from the Edg family.J Biol Chem. 2003;278(28):25600-25606.

8. Yanagida $\mathrm{K}$, et al. Identification and characterization of a novel lysophosphatidic acid receptor, p2y5/ LPA6. Jiol Chem. 2009;284(26):17731-17741.

9. Yanagida K, Ishii S, Hamano F, Noguchi K, Shimizu T. LPA4/p2y9/GPR23 mediates rho-dependent morphological changes in a rat neuronal cell line. J Biol Chem. 2007;282(8):5814-5824.

10. Thumkeo D, Watanabe S, Narumiya S. Physiological roles of Rho and Rho effectors in mammals. Eur J Cell Biol. 2013;92(10-11):303-315.

11. Lee CW, Rivera R, Gardell S, Dubin AE, Chun J. GPR92 as a new G12/13- and Gq-coupled lysophosphatidic acid receptor that increases cAMP, LPA5. J Biol Chem. 2006;281(33):23589-23597.

12. Tanaka M, et al. Autotaxin stabilizes blood vessels and is required for embryonic vasculature by producing lysophosphatidic acid. J Biol Chem. 2006;281(35):25822-25830.

13. Offermanns S, Mancino V, Revel JP, Simon MI. Vascular system defects and impaired cell chemokinesis as a result of G $\alpha 13$ deficiency. Science. 1997;275(5299):533-536.

14. Kamijo H, et al. Impaired vascular remodeling in the yolk sac of embryos deficient in ROCK-I and ROCK-II. Genes Cells. 2011;16(10):1012-1021.

15. Hata E, et al. Lysophosphatidic acid receptors LPA4 and LPA6 differentially promote lymphocyte transmigration across high endothelial venules in lymph nodes. Int Immunol. 2016;28(6):283-292.

16. Sumida $\mathrm{H}$, et al. LPA4 regulates blood and lymphatic vessel formation during mouse embryogenesis. Blood. 2010;116(23):5060-5070.

17. Roca C, Adams RH. Regulation of vascular morphogenesis by Notch signaling. Genes Dev. 2007;21(20):2511-2524.

18. Siebel C, Lendahl U. Notch signaling in development, tissue homeostasis, and disease. Physiol Rev. 2017;97(4):1235-1294.

19. Iso T, Hamamori Y, Kedes L. Notch signaling in vascular development. Arterioscler Thromb Vasc Biol. 2003;23(4):543-553.

20. Hellström M, et al. Dll4 signalling through Notch1 regulates formation of tip cells during angiogenesis. Nature. 2007;445(7129):776-780.

21. Sacilotto N, et al. Analysis of Dll4 regulation reveals a combinatorial role for Sox and Notch in arterial development. Proc Natl Acad Sci U S A. 2013;110(29):11893-11898.

22. Shah AV, et al. The endothelial transcription factor ERG mediates Angiopoietin-1-dependent control of Notch signalling and vascular stability. Nat Commun. 2017;8:16002.

23 . Corada $M$, et al. The Wnt $/ \beta$-catenin pathway modulates vascular remodeling and specification by upregulating Dll4/Notch signaling. Dev Cell. 2010;18(6):938-949.

24. Lobov IB, et al. Delta-like ligand 4 (Dll4) is induced by VEGF as a negative regulator of angiogenic sprouting. Proc Natl Acad Sci U S A. 2007;104(9):3219-3224.

25. Duarte A, et al. Dosage-sensitive requirement for mouse Dll4 in artery development. Genes Dev. 2004;18(20):2474-2478.

26. de la Pompa JL, Epstein JA. Coordinating tissue interactions: Notch signaling in cardiac development and disease. Dev Cell. 2012;22(2):244-254.

27. Trindade A, et al. Overexpression of delta-like 4 induces arterialization and attenuates vessel formation in developing mouse embryos. Blood. 2008;112(5):1720-1729.

28. Piccolo S, Dupont S, Cordenonsi M. The biology of YAP/TAZ: hippo signaling and beyond. Physiol Rev. 2014;94(4):1287-1312.

29. Dupont $S$, et al. Role of YAP/TAZ in mechanotransduction. Nature. 2011;474(7350):179-183.

30. Yu FX, et al. Regulation of the Hippo-YAP pathway by G-protein-coupled receptor signaling. Cell. 2012;150(4):780-791.

31. Kim J, et al. YAP/TAZ regulates sprouting angiogenesis and vascular barrier maturation. JClin Invest. 2017;127(9):3441-3461.

32. Wang $X$, et al. YAP/TAZ orchestrate VEGF signaling during developmental angiogenesis. Dev Cell. 2017;42(5):462-478.e7.

33. Sakabe M, et al. YAP/TAZ-CDC42 signaling regulates vascular tip cell migration. Proc Natl Acad Sci U S A. 2017;114(41):10918-10923.

34. Choi HJ, et al. Yes-associated protein regulates endothelial cell contact-mediated expression of angiopoietin-2. Nat Commun. 2015;6:6943.

35. He J, et al. Yes-associated protein promotes angiogenesis via signal transducer and activator of transcription 3 in endothelial cells. Circ Res. 2018;122(4):591-605.

36. Neto F, et al. YAP and TAZ regulate adherens junction dynamics and endothelial cell distribution during vascular development. Elife. 2018;7:e31037.

37. Wang Y, et al. Ephrin-B2 controls VEGF-induced angiogenesis and lymphangiogenesis. Nature. 2010;465(7297):483-486.

38. Cheng Z, Garvin D, Paguio A, Stecha P, Wood K, Fan F. Luciferase reporter assay system for deciphering GPCR pathways. Curr Chem Genomics. 2010;4:84-91.

39. Yanagida K, et al. The Ga12/13-coupled receptor LPA4 limits proper adipose tissue expansion and remodeling in diet-induced obesity. JCI Insight. 2018;3(24):97293.

40. Ohta H, et al. Ki16425, a subtype-selective antagonist for EDG-family lysophosphatidic acid receptors. Mol Pharmacol. 2003;64(4):994-1005.

41. Nishio M, et al. Dysregulated YAP1/TAZ and TGF- $\beta$ signaling mediate hepatocarcinogenesis in Mobla/1b-deficient mice. Proc Natl Acad Sci U S A. 2016;113(1):E71-E80.

42. Nakatsu MN, Hughes CC. An optimized three-dimensional in vitro model for the analysis of angiogenesis. Methods Enzymol. 2008;443:65-82.

43. Dovey HF, et al. Functional $\gamma$-secretase inhibitors reduce $\beta$-amyloid peptide levels in brain. J Neurochem. 2001;76(1):173-181.
44. Kim M, Kim T, Johnson RL, Lim DS. Transcriptional co-repressor function of the hippo pathway transducers YAP and TAZ. Cell Rep. 2015;11(2):270-282.

45. Zhao B, et al. TEAD mediates YAP-dependent gene induction and growth control. Genes Dev. 2008;22(14):1962-1971.

46. Zhang J, et al. Angiopoietin-1/Tie2 signal augments basal Notch signal controlling vascular quiescence by inducing $\Delta$-like 4 expression through AKT-mediated activation of $\beta$-catenin. $J$ Biol Chem. 2011;286(10):8055-8066.

47. Krebs LT, et al. Notch signaling is essential for vascular morphogenesis in mice. Genes Dev. 2000;14(11):1343-1352.

48. Neves SR, Ram PT, Iyengar R. G protein pathways. Science. 2002;296(5573):1636-1639.

49. Inoue A, Arima N, Ishiguro J, Prestwich GD, Arai H, Aoki J. LPA-producing enzyme PA-PLA ${ }_{1} \alpha$ regulates hair follicle development by modulating EGFR signalling. EMBO J. 2011;30(20):4248-4260.

50. Lee M, Choi S, Halldén G, Yo SJ, Schichnes D, Aponte GW. P2Y5 is a G $(\alpha) i, G(\alpha) 12 / 13$ G protein-coupled receptor activated by lysophosphatidic acid that reduces intestinal cell adhesion. Am J Physiol Gastrointest Liver Physiol. 2009;297(4):G641-G654.

51. Burridge K, Wennerberg K. Rho and Rac take center stage. Cell. 2004;116(2):167-179.

52. Contos JJ, et al. Characterization of lpa(2) (Edg4) and lpa(1)/lpa(2) (Edg2/Edg4) lysophosphatidic acid receptor knockout mice: signaling deficits without obvious phenotypic abnormality attributable to lpa(2). Mol Cell Biol. 2002;22(19):6921-6929.

53. Hama K, et al. Lysophosphatidic acid and autotaxin stimulate cell motility of neoplastic and non-neoplastic cells through LPA1.J Biol Chem. 2004;279(17):17634-17639.

54. Mo JS, Yu FX, Gong R, Brown JH, Guan KL. Regulation of the Hippo-YAP pathway by protease-activated receptors (PARs). Genes Dev. 2012;26(19):2138-2143.

55. Griffin CT, Srinivasan Y, Zheng YW, Huang W, Coughlin SR. A role for thrombin receptor signaling in endothelial cells during embryonic development. Science. 2001;293(5535):1666-1670.

56. Allende ML, Yamashita T, Proia RL. G-protein-coupled receptor S1P1 acts within endothelial cells to regulate vascular maturation. Blood. 2003;102(10):3665-3667.

57. Wei Y, Gong J, Thimmulappa RK, Kosmider B, Biswal S, Duh EJ. Nrf2 acts cell-autonomously in endothelium to regulate tip cell formation and vascular branching. Proc Natl Acad Sci U S A. 2013;110(41):E3910-E3918.

58. Benedito R, et al. The notch ligands Dll4 and Jagged1 have opposing effects on angiogenesis. Cell. 2009;137(6):1124-1135.

59. Majumder S, et al. G-protein-coupled receptor-2-interacting protein-1 controls stalk cell fate by inhibiting $\Delta$-like 4 -Notch1 signaling. Cell Rep . 2016;17(10):2532-2541.

60. Barry ER, et al. Restriction of intestinal stem cell expansion and the regenerative response by YAP. Nature. 2013;493(7430):106-110.

61. Beyer TA, et al. Switch enhancers interpret TGF- $\beta$ and Hippo signaling to control cell fate in human embryonic stem cells. Cell Rep. 2013;5(6):1611-1624. 
62. Manderfield LJ, et al. Hippo signaling is required for Notch-dependent smooth muscle differentiation of neural crest. Development. 2015;142(17):2962-2971.

63. Pyne S, Long JS, Ktistakis NT, Pyne NJ. Lipid phosphate phosphatases and lipid phosphate signalling. Biochem Soc Trans. 2005;33(pt 6):1370-1374.

64. Yukiura H, Kano K, Kise R, Inoue A, Aoki J. LPP3 localizes LPA6 signalling to non-contact sites in endothelial cells. JCell Sci. 2015;128(21):3871-3877.

65. Wang L, et al. Integrin-YAP/TAZ-JNK cascade mediates atheroprotective effect of unidirectional shear flow. Nature. 2016;540(7634):579-582.

66. Blanco R, Gerhardt H. VEGF and Notch in tip and stalk cell selection. Cold Spring Harb Perspect Med. 2013;3(1):a006569.

67. Gerhardt H, et al. VEGF guides angiogenic sprouting utilizing endothelial tip cell filopodia.
JCell Biol. 2003;161(6):1163-1177.

68. Caolo V, et al. Feed-forward signaling by membrane-bound ligand receptor circuit: the case of NOTCH DELTA-like 4 ligand in endothelial cells. J Biol Chem. 2010;285(52):40681-40689.

69. Hsueh YJ, Chen HC, Wu SE, Wang TK, Chen JK, Ma DH. Lysophosphatidic acid induces YAP-promoted proliferation of human corneal endothelial cells via PI3K and ROCK pathways. Mol Ther Methods Clin Dev. 2015;2:15014.

70. Nakagawa Y, et al. Hyperlipidemia and hepatitis in liver-specific CREB3L3 knockout mice generated using a one-step CRISPR/Cas9 system. Sci Rep. 2016;6:27857.

71. Cong L, et al. Multiplex genome engineering using CRISPR/Cas systems. Science. 2013;339(6121):819-823.

72. Mizuno S, et al. Simple generation of albino
C57BL/6J mice with G291T mutation in the tyrosinase gene by the CRISPR/Cas9 system. Mamm Genome. 2014;25(7-8):327-334.

73. Gordon JW, Ruddle FH. Integration and stable germ line transmission of genes injected into mouse pronuclei. Science. 1981;214(4526):1244-1246.

74. Tsukahara R, et al. LPA5 signaling is involved in multiple sclerosis-mediated neuropathic pain in the cuprizone mouse model. J Pharmacol Sci. 2018;136(2):93-96.

75. Kisanuki YY, Hammer RE, Miyazaki J, Williams SC, Richardson JA, Yanagisawa M. Tie2-Cre transgenic mice: a new model for endothelial cell-lineage analysis in vivo. Dev Biol. 2001;230(2):230-242.

76. Larrivée B, et al. ALK1 signaling inhibits angiogenesis by cooperating with the Notch pathway. Dev Cell. 2012;22(3):489-500. 